\title{
Solutions des équations de Navier-Stokes incompressibles dans un domaine exterieur
}

Nicolas Depauw

\section{Introduction.}

Le mouvement d'un fluide incompressible visqueux, de viscosité $\varepsilon$, remplissant un ouvert $\Omega \subset \mathbb{R}^{n}$ à bord $\partial \Omega$ régulier est modélisé par les équations de Navier-Stokes

$$
\begin{cases}\partial_{t} u+(u \cdot \nabla) u-\varepsilon \Delta u+\nabla p=f, & \left.u\right|_{\partial \Omega}=0, \\ \nabla \cdot u=0, & \left.u\right|_{t=0}=u_{0} .\end{cases}
$$

La dimension est $n \geq 3$. Les inconnues sont le champ vectoriel de vitesses $u(t, x) \in \mathbb{R}^{n}$ et le champ scalaire pression $p(t, x) \in \mathbb{R} ;$ les variables sont $t \in \mathbb{R}$ et $x \in \Omega$. $\nabla$ est l'opérateur différentiel $\left(\partial_{x_{1}}, \ldots, \partial_{x_{n}}\right)$, noté comme un vecteur. Ainsi $\nabla \cdot u$ est la divergence du champ $u$, tandis que $(u \cdot \nabla)$ est l'opérateur de dérivation partielle $u_{1} \partial_{x_{1}}+\cdots+u_{n} \partial_{x_{n}}$. $\Delta$ est l'opérateur de Laplace $(\nabla \cdot \nabla)$.

L'équation de Navier-Stokes présente deux types de difficultés. D'une part elle est non linéaire. Dans la résolution de cette équation, le terme $(u \cdot \nabla) u$ est souvent traité comme un terme de perturbation. D'autre part, en ignorant le terme non linéaire, cette équation ressemble beaucoup à l'équation de la chaleur avec condition de Dirichlet

$$
\begin{cases}\partial_{t} u-\varepsilon \Delta u=0=f, & \left.u\right|_{\partial \Omega}=0 \\ & \left.u\right|_{t=0}=u_{0}\end{cases}
$$


mais en diffère par l'incompressibilité. Prenant modèle sur l'équation de la chaleur, on peut résoudre l'équation de Stokes avec second membre

$$
\begin{cases}\partial_{t} u-\varepsilon \Delta u+\nabla p=f, & \left.u\right|_{\partial \Omega}=0, \\ \nabla \cdot u=0, & \left.u\right|_{t=0}=u_{0},\end{cases}
$$

à l'aide d'un semi-groupe d'opérateur, après avoir défini correctement les espaces de champs de vitesses à divergence nulle et tangent au bord.

Dans le cas de $\mathbb{R}^{n}$, de $\Omega_{b}$ borné, du demi-espace $\mathbb{R}_{+}^{n}$ et enfin d'un domaine extérieur $\Omega_{e}$, c'est-à-dire le complémentaire d'un compact, il a été démontré que le semi-groupe en question est analytique et borné uniformément en temps sur des espaces construits à partir d'espaces de Lebesgue $L^{p}(\Omega)$.

Pour traiter de la perturbation non linéaire, on la considère comme un second membre et on résout l'équation de point fixe qui en résulte par le théorème du point fixe dans les espaces de Banach. Afin d'en vérifier les hypothèses, Fujita et Kato [6] ont introduits les puissances fractionnaires du générateur du semi-groupe considéré sur $L^{2}$ et étudié leurs domaines. D'un autre côté Weissler [32] a considéré le semi-groupe sur les espaces $L^{p}$. Les différentes adaptations aux quatre cas $\left(\mathbb{R}^{n}, \Omega_{b}\right.$, $\mathbb{R}_{+}^{n}$ et $\Omega_{e}$ ) de ces techniques de semi-groupe, puissances fractionnaires et espaces de Lebesgue ont été poursuivis, entre autre grâce aux travaux de Giga [9].

Dans le cas de l'équation de Navier-Stokes sur $\mathbb{R}^{n}$, Cannone [4] a étudié l'existence globale de solutions à données petites dans l'espace de Besov homogène $\dot{B}_{p \infty}^{-1+n / p}\left(\mathbb{R}^{n}\right)$. Ce type d'argument semble avoir été poussé au maximum par Kozono et Yamazaki dans [18]. Cette étude est justifiée parce qu'une donnée initiale peut être à la fois petite dans $\dot{B}_{p \infty}^{-1+n / p}\left(\mathbb{R}^{n}\right)$ et grande dans $L^{n}\left(\mathbb{R}^{n}\right)$ à condition d'être suffisamment oscillante. De plus, l'existence de solutions à données dans de tels espaces de Besov est essentielle pour l'étude des solutions autosimilaires de l'équation de Navier-Stokes telle que l'ont menée Cannone et Planchon [27], [5], [28]. Les méthodes utilisées par ces auteurs reposent de manière essentielle sur l'expression explicite du noyau de la chaleur par transformation de Fourier.

Nous exposons dans cet article l'analogue de ces résultats d'existence pour l'équation de Navier-Stokes, mais sur un domaine extérieur $\Omega_{e}$, complémentaire d'un compact à bord lisse. Les deux difficultés nouvelles qui se présentent sont l'absence d'une représentation explicite en Fourier du semi-groupe associé à l'opérateur de Stokes et la nécessité de transposer la notion d'espace de Besov homogène. 
La méthode de point fixe utilisée depuis Weissler, reprise par Kato [16] et plus récemment par Cannone et Planchon, présente le défaut de n'assurer l'unicité d'une solution continue à valeur $L^{n}\left(\mathbb{R}^{n}\right)$, pour une donnée initiale dans $L^{n}$ petite dans $\dot{B}_{p \infty}^{-1+n / p}$, que dans une boule d'un sous-espace de l'ensemble $C\left(L^{n}\left(\mathbb{R}^{n}\right)\right)$ des fonctions continue de $[0, \infty[$ dans $L^{n}\left(\mathbb{R}^{n}\right)$.

Récemment, Furioli, Lemarie-Rieusset et Terraneo [7], [8] ont obtenu un remarquable résultat d'unicité des solutions locales $C\left(L^{3}\left(\mathbb{R}^{3}\right)\right)$, à l'aide de ces espaces de Besov homogènes. Signalons au passage que Lions et Masmoudi [21] ont annoncé une autre démonstration, encore valable dans le cas d'un domaine $\Omega$ à bord régulier.

Pour le cas de $\Omega_{e}$ domaine extérieur, nous retrouvons le résultat d'unicité des solutions locales $C\left(L^{n}(\Omega)\right)$. Nous preferrons utiliser là les espaces de Besov non homogènes de Kobayashi et Muramutu [17], pour éviter la détérioration de l'estimation du gradient du semi-groupe en temps grand quand $\Omega=\Omega_{e}$ mise en évidence par Maremonti et Solonnikov [22].

Un certain nombre d'auteurs ont utilisé des espaces de Besov non homogènes sur un domaine extérieur. Grubb et Solonnikov [13], [12] ont introduit de tels espaces pour résoudre sur $I \times \Omega_{b}$ ( $I$ intervalle de $\mathbb{R}, \Omega_{b}$ ouvert borné) les équations de Navier-Stokes avec toute une variété de conditions au bord et un second membre. Il s'agit d'espaces de Besov avec des régularités différentes en temps et en espace, ce qui donne des résultats très précis sur les conditions de compatibilité que doivent vérifier les données pour obtenir existence et unicité de solutions régulières. Grubb [11] a récemment adapté cette méthode au cas de $\Omega_{e}$ domaine extérieur, mais en n'utilisant que des espaces non homogènes, son résultat n'est que local en temps. De mème pour Kobayashi et Muramutu [17] qui ont obtenu sur $\Omega_{e}$ un résultat d'existence locale en temps pour une donnée initiale dans un espace de Besov abstrait non homogène construit par interpolation réelle à partir du générateur du semi-groupe. Encore pour $\Omega_{e}$, mentionnons que Borchers et Myakawa [2] avaient utilisé des espaces d'interpolation complexe définis à partir du générateur du semi-groupe (qui sont en quelque sorte l'analogue des espaces de Bessel homogènes) pour obtenir des estimations cœrcives homogènes optimales.

Pour les espaces homogènes, signalons en plus de [4] et [27], que Kozono et Yamazaki dans [19] présentent un résultat d'existence globale à donnée petite dans l'espace de Lorentz $L^{n \infty}\left(\Omega_{e}\right)$ pour un domaine $\Omega$, par la méthode de Kato. Meyer dans [24] donne des résultats 
de continuité pour le terme non-linéaire dans $L^{n \infty}\left(\mathbb{R}^{n}\right)$, qui sont faux dans $L^{3}\left(\mathbb{R}^{3}\right)$ d'après Oru [26], et qui permettent tout à la fois de prouver l'existence globale à donnée petite dans $L^{n \infty}\left(\mathbb{R}^{n}\right)$ et de retrouver l'unicité des solutions $C\left(L^{3}\left(\mathbb{R}^{3}\right)\right)$.

Plan DE L'ARTiCle. Dans les préliminaires, après des notations générales nous étudions l'opérateur de Stokes, son semi-groupe associé, puis décrivons les espaces fonctionnels construits avec. La section suivante rassemble les énoncés des résultats importants de l'article : existence à donnée petite, avec un exemple, et unicité. La troisième section est consacrée à la démonstration des théorèmes, après étude de la continuité du terme non linéaire. La dernière section expose en détail l'exemple.

\section{Préliminaires.}

\subsection{Notations générales.}

On note $\left.\mathbb{R}_{+} \doteq\right] 0, \infty\left[\right.$ et $\overline{\mathbb{R}}_{+} \doteq\left[0, \infty\left[. C(I ; X)\right.\right.$ et $C_{b}(I ; X)$ désignent respectivement les fonctions continues et continues bornées de $I$ dans $X$.

Notons $\mathcal{L}(X ; Y)$ l'espace de Banach des applications linéaires continues d'un espace de Banach $X$ dans un espace de Banach $Y$, et $\|T\|_{\mathcal{L}(X ; Y)}$ la norme d'un opérateur $T$ élément de cet espace. Si $X=Y$, on écrit seulement $\mathcal{L}(X)$. Si $X$ est inclus dans $Y$ et si l'injection est continue, on écrit $X \hookrightarrow Y$.

Nous considérons un ouvert $\Omega$ de $\mathbb{R}^{n}$ dont le bord $\partial \Omega$ est lisse et dont le complémentaire $K$ est compact. Pour $p \in] 1, \infty[$ et $k \in \mathbb{N}$, on note $W_{p}^{k}(\Omega)$ l'espace de Sobolev des distributions dont les dérivées jusqu'à l'ordre $k$ sont dans $L^{p}(\Omega)$, et $W_{p, 0}^{k}(\Omega)$ l'adhérence dans $W_{p}^{k}(\Omega)$ des fonctions test $C_{0}^{\infty}(\Omega)$. On note $L_{\mathrm{loc}}^{p}(\Omega)$ les distributions sur $\Omega$ dont la restriction à $B \cap \Omega$, pour toute boule $B$ de $\mathbb{R}^{n}$, est dans $L^{p}(B \cap \Omega)$. On note $\nabla^{k} u$ le gradient itéré $k$ fois d'une distribution, c'est-à-dire la collection des $\partial_{x}^{\alpha} u=\partial_{x_{\alpha_{1}}} \cdots \partial_{x_{\alpha_{k}}} u$ pour $\alpha$ décrivant l'ensemble des applications de $\{1, \ldots, k\}$ dans $\{1, \ldots, n\}$, de sorte que, par exemple, $\sum_{r=0}^{k}\left\|\nabla^{r} u\right\|_{p}$ est une norme équivalente sur $W_{p}^{k}(\Omega)$.

Nous aurons aussi besoin quelquefois :

- des espaces de Lorentz $L^{p q}(\Omega)$ pour $q \in[1, \infty]$, obtenus par interpolation réelle à partir des $L^{p}(\Omega)$; 
- des espaces de Besov $B_{p q}^{s}(\Omega)$ pour $s \in \mathbb{R}_{+} \backslash \mathbb{N}$ et $q \in[1, \infty]$, obtenus par interpolation réelle à partir des $W_{p}^{k}(\Omega)$;

- des espaces de Sobolev sur le bord $W_{p}^{k}(\partial \Omega), k \in \mathbb{N}$ et même des espaces de Slobodetskii $W_{p}^{s}(\partial \Omega)$ (dits aussi espaces de traces) pour $s \in \mathbb{R}_{+} \backslash \mathbb{N}$ obtenus par interpolation réelle à partir des précédents.

Il est possible d'étendre ces définitions aux indices $k \in \mathbb{Z}$ et $s \in \mathbb{R}$ (voir [30]).

On note $\nu$ le vecteur normal (unitaire rentrant) au bord de $\Omega$ et $\partial_{\nu}$ l'opérateur différentiel associé. On note $\gamma_{0} u$ la restriction à $\partial \Omega$ d'une fonction continue sur $\bar{\Omega}$ et $\gamma_{1} u \doteq \gamma_{0} \partial_{\nu} u$ si $u$ est continûment dérivable. On sait étendre l'action de ces opérateurs à certains espaces de distributions. Par exemple $\gamma_{0}$ est continu de $W_{p}^{1}(\Omega)$ dans $W_{p}^{1-1 / p}(\partial \Omega)$, mais n'est pas continu sur $L^{p}(\Omega)$. Pour une distribution $u$ sur $\partial \Omega$ à valeur vectorielle, on définit l'opérateur de projection orthogonale $\pi_{\nu} u=(u \cdot \nu)$ sur $\nu$. On note $\gamma_{\nu} \doteq \pi_{\nu} \gamma_{0}$ l'opérateur de trace normale au bord.

On note $X_{p}$ (respectivement $X_{p q}$ ) l'adhérence dans $L^{p}(\Omega)$ (respectivement $L^{p q}(\Omega)$ ) des champs de vecteurs $C_{0}^{\infty}(\Omega)$ à divergence nulle. Il est bien connu que $X_{p}$ coïncide avec le sous-espace fermé des $u \in L^{p}(\Omega)$ tels que $\nabla \cdot u=0$ et $\gamma_{\nu} u=0$. Ici $\nabla$. désigne l'opérateur différentiel de divergence. La nullité de la divergence permet d'étendre l'opérateur de trace normale $\gamma_{\nu}$, au moyen d'une intégration par partie, en un opérateur continu du sous-espace fermé de $L^{p}(\Omega)$ d'équation $\nabla \cdot u=0$ dans $W_{p}^{-1 / p}(\partial \Omega)$. On sait aussi qu'on peut décomposer en somme directe l'espace de Banach

$$
L^{p}(\Omega)=X_{p} \oplus\left\{\nabla p \in L^{p}(\Omega): p \in L_{\mathrm{loc}}^{p}(\Omega)\right\}
$$

et qu'il existe un opérateur linéaire $P$, continu pour tout $1<p<\infty$ sur les champs de vecteurs $L^{p}(\Omega)$, qui est une projection (en particulier $P^{2}=P$ ) sur $P L^{p}(\Omega)=X_{p}$ parallèlement aux champs gradients, orthogonale pour la structure euclidienne de $L^{2}(\Omega)$. Pour le cas relativement général qui nous intéresse, à savoir $1<p<\infty$ et $\Omega$ non borné, mais cependant $\partial \Omega$ compact, la preuve de ces trois affirmations est présentée en détail dans [25].

On note $p^{\prime}$ l'exposant conjugué de $p$ défini par $1 / p+1 / p^{\prime}=1$. On peut identifier $X_{p^{\prime}}$ au dual de $X_{p}$, qui est donc réflexif. L'adjoint du projecteur $P: L^{p}(\Omega) \longrightarrow L^{p}(\Omega)$ est le projecteur $P: L^{p^{\prime}}(\Omega) \longrightarrow$ $L^{p^{\prime}}(\Omega)$, tandis que si on considère $P: L^{p}(\Omega) \longrightarrow X_{p}$, son adjoint est simplement l'injection canonique $I: X_{p^{\prime}} \longrightarrow L^{p^{\prime}}(\Omega)$. 
On note $A_{p}$ l'opérateur dans $X_{p}$, de domaine

$$
\mathcal{D}\left(A_{p}\right)=W_{p}^{2} \cap W_{p, 0}^{1} \cap X_{p}
$$

et agissant comme l'opérateur de Stokes $A=-P \Delta$. On remplace alors la résolution de l'équation linéaire de Stokes pour la viscosité $\varepsilon=1$

$$
\left\{\begin{array}{l}
\partial_{t} u-\Delta u+\nabla p=0, \\
\nabla \cdot u=0, \quad \gamma_{0} u=u_{\mid \partial \Omega}=0,
\end{array}\right.
$$

par l'équation différentielle abstraite écrite dans $X_{p}$ qu'on obtient en appliquant le projecteur $P$ à l'équation de Stokes

$$
\partial_{t} u+A u=0 \text {. }
$$

\begin{tabular}{|c|c|c|c|}
\hline Espace & Norme & Référence & Remarque \\
\hline $\mathcal{A}$ & & Déf. 16 & \\
\hline$B_{p q}^{s}(A)$ & $\left\|u \mid B_{p q}^{s}\right\|$ & Déf.13, (1.11) & \\
\hline $\mathcal{B}_{r}^{q}$ & $\left\|u \mid \mathcal{B}_{r}^{q}\right\|$ & Déf. 32 & $=B_{r \infty}^{-n / q+n / r}(A)$ \\
\hline $\mathcal{B}_{r}$ & $\left\|u \mid \mathcal{B}_{r}\right\|$ & Déf. 32 & $=\mathcal{B}_{r}^{n}$ \\
\hline$\dot{B}_{p q}^{s}(A)$ & $\left\|u \mid \dot{B}_{p q}^{s}\right\|$ & Déf. 16, (1.14) & \\
\hline$\dot{\mathcal{B}}_{r}$ & $\left\|u \mid \dot{\mathcal{B}}_{r}\right\|$ & Déf. 27 & $=\dot{B}_{r \infty}^{-1+n / r}(A)$ \\
\hline$E_{p}$ & $\|u\|_{E_{p}}$ & Déf. 27 & \\
\hline$E_{p ; 0}(0)$ & & Déf. 27 & \\
\hline$F_{p}^{T}$ & $\|u\|_{F_{p}^{T}}$ & Déf. 32 & \\
\hline$G_{p}^{T}$ & $\|u\|_{G_{p}^{T}}$ & Déf. 32 & $=L_{T}^{\infty}\left(\mathcal{B}_{p}\right)$ \\
\hline $\mathcal{H}$ & $\|u\|_{\mathcal{H}}$ & $(4.4)$ & \\
\hline$L_{T}^{\infty}(X)$ & & Déf. 32 & \\
\hline$L^{p *}$ & $\|f\|_{p *}$ & Not. 11 & \\
\hline$L^{p * 0}$ & $\|f\|_{p * 0}$ & Not. 11 & \\
\hline$L^{p * \infty}$ & $\|f\|_{p * \infty}$ & Not. 11 & \\
\hline$W_{p}^{0}(A)$ & $\|u\|_{p}$ & Déf. 12 & $=X_{p}$ \\
\hline$W_{p}^{2}(A)$ & $\left\|u \mid W_{p}^{2}\right\|$ & Déf. 12 & $=\mathcal{D}\left(A_{p}\right)$ \\
\hline$W_{p}^{-2}(A)$ & $\left\|u \mid W_{p}^{-2}\right\|$ & Déf. 12 & \\
\hline
\end{tabular}

Pour la commodité du lecteur, nous avons rassemblé dans le tableau 1 tous les autres espaces de fonctions définis dans la suite de l'article.

Tableau 1. Liste des espaces fonctionnels. 


\subsection{Opérateur de Stokes.}

\subsubsection{Décomposition de la résolvante.}

Nous décrivons ici comment on peut trouver $u \in X_{p}$ solution du problème de Stokes $(\lambda+A) u=f$. Précisément : étant donné $\lambda \in$ $\mathbb{C} \backslash]-\infty, 0]$ et $f \in X_{p}$, on cherche $u$ tel que

$$
(\lambda-\Delta) u+\nabla p=f, \quad \nabla \cdot u=0, \quad \gamma_{0} u=0 .
$$

Nous suivons les exposés de [9], [25].

Soit $f \in X_{p}$. On note $\tilde{f} \in L^{p}\left(\mathbb{R}^{n}\right)$ son prolongement par zéro hors de $\Omega$. On voit facilement que la distribution $\nabla \cdot \widetilde{f}$ est nulle parce que $\nabla \cdot f$ et $\gamma_{\nu} f$ sont nulles. On note $\widetilde{u}$ la solution du problème de Stokes dans $\mathbb{R}^{n}$

$$
(\lambda-\Delta) \widetilde{u}+\nabla \widetilde{p}=\tilde{f}, \quad \nabla \cdot \widetilde{u}=0 .
$$

Puisque $\nabla \cdot \widetilde{f}=0$, en fait $\widetilde{p}=0$ et on peut calculer $\widetilde{u}=\widetilde{E}_{\lambda} \widetilde{f}$ au moyen de l'opérateur $\widetilde{E}_{\lambda}$ de convolution par le potentiel volume $\widetilde{e}_{\lambda}$ sur $\mathbb{R}^{n}$ qui est la transformée de Fourier inverse de $\xi \longmapsto\left(\lambda+|\xi|^{2}\right)^{-1}$. Par le théorème de Mihlin, $\widetilde{E}_{\lambda}$ est continu de $L^{p}\left(\mathbb{R}^{n}\right)$ dans $W_{p}^{2}\left(\mathbb{R}^{n}\right)$ pour chaque $1<p<\infty$.

La restriction $r_{\Omega} \widetilde{u}$ vérifie l'équation intérieure de Stokes sur $\Omega$

$$
(\lambda-\Delta) \mathrm{r}_{\Omega} \widetilde{u}=f, \quad \nabla \cdot \mathrm{r}_{\Omega} \widetilde{u}=0
$$

puisque $\mathrm{r}_{\Omega} \widetilde{p}=0$, mais pas la condition au bord. On élimine la composante normale $\gamma_{\nu}=\pi_{\nu} \gamma_{0}=\nu \cdot \gamma_{0}$ au moyen du projecteur de Leray $P$. On sait que pour $v$ dans $L^{p}(\Omega)$ à divergence nulle, la projection $P v$ s'exprime au moyen de l'opérateur solution du problème de Neumann. Fixons les notations. Pour $\phi \in W_{p}^{-1 / p}(\partial \Omega)$ avec $\int_{\partial \Omega} \phi=0$, on note $w=N \phi$ la solution du problème

$$
\left\{\begin{array}{l}
\Delta w=0, \quad \nabla w \in L^{p}(\Omega), \\
\gamma_{1} w=\phi, \quad \lim _{|x| \rightarrow \infty}|w(x)|=0
\end{array}\right.
$$

obtenue au moyen du potentiel simple couche associé à l'opérateur de Laplace $\Delta$. Voir [25] et, en annexe, la définition du potentiel simple couche et son application au problème de Neumann à la Section B.3. Remarquons que $\nabla \cdot \nabla=\Delta$ et $\gamma_{\nu} \nabla=\gamma_{1}$. L'opérateur $\nabla N$ est continu 
du sous-espace fermé de $W_{p}^{-1 / p}(\partial \Omega)$ d'équation $\int_{\partial \Omega} \phi=0$ dans $L^{p}(\Omega)$. La restriction $P_{\nu}$ de $P$ aux champs à divergence nulle s'écrit $P_{\nu}=$ $1-\nabla N \gamma_{\nu}$.

Ainsi $u_{\nu}=P \mathrm{r}_{\Omega} \widetilde{u}$ est solution de

$$
\left\{\begin{array}{l}
(\lambda-\Delta) u_{\nu}+\nabla p_{\nu}=f, \quad \nabla \cdot u_{\nu}=0, \\
\gamma_{\nu} u_{\nu}=0
\end{array}\right.
$$

avec $p_{\nu}=\lambda N \gamma_{\nu} \mathrm{r}_{\Omega} \widetilde{u}$. Évidemment la trace $\gamma_{0} u_{\nu}$ n'est pas nécessairement nulle, mais elle est tangentielle.

Soit $u_{\tau}=V_{\lambda} \psi$ la solution du problème tangentiel

$$
\begin{cases}(\lambda-\Delta) u_{\tau}+\nabla p_{\tau}=0, & \nabla \cdot u_{\tau}=0, \\ \gamma_{\nu} u_{\tau}=0, & \gamma_{0} u_{\tau}=\psi,\end{cases}
$$

avec $\psi$ un champ de vecteurs tangentiel au dessus de $\partial \Omega$. Le fait qu'on puisse définir $\gamma_{0} u_{\tau}$ dans $W_{p}^{-1 / p}(\partial \Omega)$ pour un $u$ dans $L^{p}(\Omega)$ qui vérifie les trois autres équations de (1.3) est justifié par un argument de dualité dans [9]. D'après [9, Proposition 2.2] et le théorème du graphe fermé, $V_{\lambda}$ est continu de $W_{p}^{-1 / p}(\partial \Omega)$ dans $L^{p}(\Omega)$.

Si on choisit $\psi=-\gamma_{0} u_{\nu}$, on vérifie enfin que $u=G_{\lambda} f=u_{\nu}+u_{\tau}$ est la solution du problème de Stokes

$$
\left\{\begin{array}{l}
(\lambda-\Delta) u+\nabla p=f, \quad \nabla \cdot u=0, \\
\gamma_{0} u=0
\end{array}\right.
$$

REMARQue 1. Les résultats de continuité rappelés ci-dessus sont vrais pour chaque $\lambda \in \mathbb{C} \backslash]-\infty, 0]$ fixé. Ils découlent des propriétés des systèmes elliptiques aux limites. L'amélioration principale due à [9] consiste en des estimations uniformes par rapport à $\lambda$.

\subsubsection{Résolvante et semi-groupe.}

Dans toute la suite, $\mathcal{V}_{\varepsilon}$ désigne, pour un $\varepsilon$ donné dans $[0, \pi]$, l'ensemble

$$
\mathcal{V}_{\varepsilon}=\left\{\lambda \in \mathbb{C} \backslash \overline{\mathbb{R}}_{-}:|\arg \lambda|<\varepsilon\right\} .
$$

Voici un résultat important dans l'étude du comportement en temps long des solutions de l'équation de Stokes. 
Théorème 3. Pour tout $p \in] 1, \infty\left[\right.$, l'opérateur $-A_{p}$ est $\pi$-sectoriel : étant donné $\left.\varepsilon^{\prime} \in\right] 0, \pi[$, il existe $C$ tel que

$$
\left\|(\lambda+A)^{-1} v\right\|_{p} \leq C|\lambda|^{-1}\|v\|_{p}, \quad \text { pour tous } \lambda \in \mathcal{V}_{\varepsilon^{\prime}}, v \in X_{p} .
$$

Ce théorème permet de construire des solutions à l'équation de Stokes au moyen du semi-groupe engendré par $-A$.

Corollaire 4 ([15, p. 487-492]). Pour tout $p \in] 1, \infty[$, l'intégrale de Dunford

$$
U(t) \doteq \frac{1}{2 i \pi} \int_{\Gamma} e^{t \lambda}(\lambda+A)^{-1} d \lambda
$$

où $\Gamma$ est un chemin dans $\mathbb{C}$ contournant $]-\infty, 0]$ dans le sens positif, de $\infty \cdot e^{-i \theta} \grave{a} \infty \cdot e^{i \theta}$ pour $\left.\theta \in\right] \pi / 2, \pi[$, définit une application $t \longmapsto U(t)$ de $\mathcal{V}_{\pi} \cup\{0\}$ dans $\mathcal{L}\left(X_{p}\right)$, indépendante de $\theta$, vérifiant sur cet ensemble la règle de composition $U(t) U(s)=U(t+s)$, holomorphe sur $\mathcal{V}_{\pi}$, et telle que pour tout $\varepsilon^{\prime}<\pi$, il existe une constante $C$ avec

$$
\begin{gathered}
\|U(t) x\|_{p} \leq C\|x\|_{p}, \quad \text { pour tous } t \in \mathcal{V}_{\varepsilon^{\prime}} \cup\{0\}, x \in X, \\
\lim _{\substack{t \rightarrow 0 \\
t \in \mathcal{V}_{\varepsilon^{\prime}}}}\|U(t) x-x\|_{p}=0, \quad \text { pour tout } x \in X
\end{gathered}
$$

Pour tout $k \in \mathbb{N}$, l'application $t \longmapsto t^{k} A^{k} U(t)$ est continue bornée de $\mathbb{R}_{+}$dans $\mathcal{L}(X)$ et $\partial_{t} U(t)=-A U(t)$.

Rappelons que le domaine $\mathcal{D}\left(A^{\prime}\right)$ du dual d'un opérateur $A$ non borné dans $X$ de domaine $\mathcal{D}(A)$ dense dans $X$ est l'ensemble des $x^{\prime} \in X^{\prime}$ tel qu'il existe $y^{\prime} \in X^{\prime}$ vérifiant pour tout $x \in \mathcal{D}(A)$ l'égalité $\left\langle x^{\prime}, A x\right\rangle=$ $\left\langle y^{\prime}, x\right\rangle$.

Théorème 5 ([25]). On a les identifications d'espaces et d'opérateurs duaux

$$
\mathcal{D}\left(A_{p}{ }^{\prime}\right)=\mathcal{D}\left(A_{p^{\prime}}\right), \quad A_{p}{ }^{\prime}=A_{p^{\prime}}
$$

Proposition 6. Soit $p$ et $q$ dans $] 1, \infty[$ tels que $0 \leq 1 / p-1 / q \leq 1 / n$. Alors l'application $t \longmapsto(1+A)^{-1} U(t) P \nabla \cdot$ est continue bornée de $\mathbb{R}_{+}$ dans $\mathcal{L}\left(L^{p}(\Omega) ; X_{q}\right)$. 
DÉmonstration. On sait déjà que $(1+A)^{-1}$ et $U(t)$ commutent et que $U(t) \in C_{b}\left(\mathbb{R}_{+} ; \mathcal{L}\left(X_{q}\right)\right)$. Montrons que $(1+A)^{-1} P \nabla \cdot \in \mathcal{L}\left(L^{p}(\Omega) ; X_{q}\right)$. Par définition de $\mathcal{D}\left(A_{q^{\prime}}\right)$ et injection de Sobolev de $W_{q^{\prime}}^{1}(\Omega)$ dans $L^{p^{\prime}}(\Omega)$ pour $0 \leq 1 / q^{\prime}-1 / p^{\prime} \leq 1 / n$, on obtient $\nabla(1+A)^{-1} \in \mathcal{L}\left(X_{q^{\prime}} ; L^{p^{\prime}}(\Omega)\right)$. Or $\nabla(1+A)^{-1}=\nabla I(1+A)^{-1}$ est bien l'opérateur dual de $(1+A)^{-1} P \nabla \cdot$.

Pour le semi-groupe engendré par l'opérateur de Stokes, on dispose d'estimations $L^{p}-L^{q}$.

Définition 7. On note

$$
[p, q]=\frac{n}{2}\left(\frac{1}{p}-\frac{1}{q}\right)
$$

Théorème 8 (Estimations $\left.L^{p}-L^{q}[2],[14],[22]\right)$.

1) Étant donné $1<p \leq q<\infty$, il existe $C$ tel que

$$
\|U(t) v\|_{q} \leq C\|v\|_{p} t^{-[p, q]}, \quad \text { pour tout } v \in X_{p} .
$$

2) Étant donné $1<p \leq q \leq n$, il existe $C$ tel que

$$
\|\nabla U(t) v\|_{q} \leq C\|v\|_{p} t^{-1 / 2-[p, q]}, \quad \text { pour tout } v \in X_{p} .
$$

3) Étant donné $1<p \leq q<\infty$ avec $n \leq q$, il existe $C$ tel que

$$
\|\nabla U(t) v\|_{q} \leq C\|v\|_{p} t^{-1 / 2-[p, q]}(1+t)^{[n, q]}, \quad \text { pour tout } v \in X_{p} .
$$

Remarque 9 . Dans le cas de $\mathbb{R}^{n},(1.7)$ est valable pour tout $p, q$ dans ] $1, \infty[$ avec $p \leq q$. [16] en déduit un théorème d'existence globale en temps d'une solution à l'équation de Navier-Stokes dans $C\left(L^{n}\left(\mathbb{R}^{n}\right)\right)$ quand la donnée initiale est suffisamment petite dans $L^{n}$.

Pour le domaine extérieur $\Omega$, le cas limite $n=p$ est atteint d'après [14]. Cela lui permet d'étendre le résultat de [16] à ce cas. [22] montre que (1.8) est optimale. Notons que pour $t$ grand, l'exposant est

$$
-\frac{1}{2}-[p, q]+[n, q]=-\frac{n}{2 p} \text {. }
$$

Corollaire 10. Soit $p$ et $q$ dans $] 1, \infty[$ tels que $p \leq q$, et $k$ dans $\mathbb{N}$. 
1) Si $n^{\prime} \leq p$, alors l'application $t \longmapsto t^{k+1 / 2+[p, q]} A^{k} U(t) P \nabla \cdot$ est continue bornée de $\mathbb{R}_{+}$dans $\mathcal{L}\left(L^{p}(\Omega) ; X_{q}\right)$.

2) Pour tout $T>0$, l'application $t \longmapsto t^{k+1 / 2+[p, q]} A^{k} U(t) P \nabla \cdot$ est continue bornée de $] 0, T\left[\right.$ dans $\mathcal{L}\left(L^{p}(\Omega) ; X_{q}\right)$.

DÉmonstration. Considérons d'abord le premier point, cas $k=0$. On procède par dualité comme pour la Proposition 6. L'opérateur dual de $\nabla U(t)=\nabla I U(t)$ est $U(t) P \nabla \cdot$. La borne dans $\mathcal{L}\left(L^{p}(\Omega) ; X_{q}\right)$ vient de (1.7). Pour $k>0$, on écrit $A^{k} U(t) P \nabla \cdot=\left(A^{k} U(t / 2)\right)(U(t / 2) P \nabla \cdot)$, puis on applique le cas $k=0$ à $U(t / 2) P \nabla \cdot$ et le Corollaire 4 à $A^{k} U(t / 2)$ Le second point est très semblable : on utilise (1.8) et la borne sur $t$. La continuité vient du Corollaire 4.

\subsection{Description des espaces fonctionnels.}

Notation 11. On munit $\mathbb{R}_{+}$de la mesure de Haar $d t / t$ associée à la structure de groupe multiplicatif. Alors on note $L^{p *}$ l'espace des fonctions de puissance $p$-ième intégrable, avec la norme

$$
\|f\|_{p *} \doteq\left(\int_{0}^{\infty}|f(\tau)|^{p} \frac{d \tau}{\tau}\right)^{1 / p}
$$

pour $1 \leq p<\infty$. Pour $p=\infty$, l'extension usuelle de la définition coïncide avec $L^{\infty}\left(\mathbb{R}_{+}\right)$. Si on considère seulement l'intervalle ]0,1] (respectivement $\left[1, \infty\left[\right.\right.$ ), on notera respectivement $L^{p * 0}$ et $\|f\|_{p * 0}$ (respectivement $L^{p * \infty}$ et $\left.\|f\|_{p * \infty}\right)$.

Si $f$ est à valeur dans un espace de Banach $(X,\|\cdot\|)$, on se place dans le cadre de la théorie de l'intégrale de Bochner en supposant que $f$ est fortement mesurable. D'après le théorème de Pettis, il suffit que $f$ soit faiblement mesurable (i.e. pour tout $f^{\prime} \in X^{\prime}, s \longmapsto\left\langle f^{\prime}, f(s)\right\rangle$ est mesurable) et presque partout à valeur séparable (i.e. il existe un ensemble $S$ de mesure nulle telle que $f\left(\mathbb{R}_{+} \backslash S\right)$ soit séparable).

On rappelle en Annexe A la définition et les propriétés principales de l'interpollation réelle $\left(A_{0}, A_{1}\right)_{\theta, \infty}$ entre deux espaces de Banach $A_{0}$ et $A_{1}$. 


\subsubsection{Extrapolation.}

Définition 12. Notons $W_{p}^{0}(A) \doteq X_{p}$, et $W_{p}^{2}(A) \doteq \mathcal{D}\left(A_{p}\right)$ le domaine de l'opérateur de Stokes défini par (1.2), muni de la norme $\left\|u \mid W_{p}^{2}\right\| \doteq$ $\|(1+A) u\|_{p}$. Comme dans [17], on définit aussi des espaces d'indice négatif : $W_{p}^{-2}(A)$ est l'espace complété à partir de $X_{p}$ pour la norme $\left\|u \mid W_{p}^{-2}\right\| \stackrel{p}{=}\left\|(1+A)^{-1} u\right\|_{p}$.

Puisque le graphe $\mathcal{G}_{A_{p}}$ est fermé et que le domaine $\mathcal{D}\left(A_{p}\right)$ est dense dans $X_{p}$, cet espace coïncide avec la construction plus abstraite par quotient : $\left(X_{p} \times X_{p}\right) / \mathcal{G}_{A_{p}}$, introduite dans [10]. Ceci est expliqué en détail dans [31]. On y apprend aussi qu'en lien avec la dualité (1.5), cet espace est le dual de $\mathcal{D}\left(A_{p^{\prime}}\right)$.

On peut étendre $A$ en un opérateur non borné dans $W_{p}^{-2}(A)$, fermé de domaine $W_{p}^{0}(A)$ dense. Il hérite des propriétés spectrales de l'opérateur $A$ dans $X_{p}$. On peut aussi étendre à $W_{p}^{-2}(A)$ tout opérateur pris dans $\mathcal{L}\left(X_{p}\right)$ qui commute avec la résolvante $(1+A)^{-1}$. En particulier $U(t)$ admet une telle extension, qui coïncide avec le semi-groupe engendré par l'extension de $A$. Le domaine du carré de l'extension de $A$ est $W_{p}^{2}(A)$. Pour tout $t>0$, l'opérateur $U(t)$ est continu de $W_{p}^{-2}(A)$ dans $W_{p}^{2}(A)(c f .[17])$.

\subsubsection{Espaces de Besov.} réelle.

Comme $W_{p}^{2}(A) \hookrightarrow W_{p}^{-2}(A)$, on peut leur appliquer l'interpolation

Définition 13. Pour $s=-2(1-\theta)+2 \theta$, avec $0<\theta<1$ et donc $|s|<2$, pour $p \in] 1, \infty[$ et $q \in[1, \infty]$

$$
B_{p q}^{s}(A) \doteq\left(W_{p}^{-2}(A), W_{p}^{2}(A)\right)_{\theta, q}
$$

Il est bien connu (voir [20]) que

$$
W_{p}^{2}(A) \hookrightarrow B_{p q}^{s}(A) \hookrightarrow W_{p}^{-2}(A) .
$$

De plus on peut montrer (voir [10]) que $B_{p 1}^{0}(A) \hookrightarrow W_{p}^{0}(A) \hookrightarrow B_{p \infty}^{0}(A)$. Du théorème de réitération, il vient alors $B_{p q}^{s}(A)=\left(W_{p}^{0}(A), W_{p}^{2}(A)\right)_{\theta, q}$ pour $s=2 \theta \in] 0,2[$. 
[10] donne une expression de la norme dans ces espaces à l'aide des puissances de la résolvante $(\lambda+A)^{-n}$. On trouve dans [17] la définition équivalente suivante, valable pour $|s|<2$

$$
B_{p q}^{s}(A)=\left\{u \in W_{p}^{-2}(A):\left\|\lambda^{s / 2}\right\| \lambda A(\lambda+A)^{-2} u\left\|_{p}\right\|_{q * \infty}<\infty\right\}
$$

muni de la norme

$$
\left\|u \mid W_{p}^{-2}\right\|+\left\|\lambda^{s / 2}\right\| \lambda A(\lambda+A)^{-2} u\left\|_{p}\right\|_{q * \infty} .
$$

Le lien avec les solutions de l'équation d'évolution est plus sensible quand on exprime ces normes avec le semi-groupe engendré par l'opérateur.

Lemme 14. Pour $|s|<2, B_{p q}^{s}(A)$ est l'ensemble des éléments de $W_{p}^{-2}(A)$ tels que la quantité

$$
\left\|u \mid B_{p q}^{s}\right\| \doteq\|U(1) u\|_{p}+\left\|t^{-s / 2}\right\| t A U(t) u\left\|_{p}\right\|_{q * 0}
$$

soit finie. Cette quantité définit alors une norme équivalente.

DÉmonstration. Notons provisoirement $\|u\|_{X}$ la quantité définie par (1.11), et $X$ l'ensemble des éléments de $W_{p}^{-2}(A)$ qui vérifient $\|u\|_{X}<$ $\infty$.

Montrons d'abord $B_{p q}^{s}(A) \hookrightarrow X$. Soit $u \in B_{p q}^{s}(A)$. On peut écrire (voir Proposition 48) pour $\tau \in \mathbb{R}_{+}$,

$$
u=u_{-}(\tau)+u_{+}(\tau)
$$

avec, quand on a fixé $s_{-}$et $s_{+}$deux réels tels que

$$
s_{-} s_{+}<0, \quad(1-\theta) s_{-}+\theta s_{+}=0,
$$

comme définition d'une norme équivalente

$$
\left\|u \mid B_{p q}^{s}\right\|=\inf \left(\left\|\tau^{s_{-}}\right\| u_{-}(\tau)\left|W_{p}^{-2}\|\|_{q *}+\left\|\tau^{s_{+}}\right\| u_{+}(\tau)\right| W_{p}^{2}\|\|_{q_{*}}\right)
$$

où la borne inférieure est prise sur toutes les décompositions (1.12). Or $U(1) \in \mathcal{L}\left(W_{p}^{-2}(A) ; W_{p}^{0}(A)\right)$ donc (1.9) implique $\|U(1) u\|_{p} \leq C \| u \mid$ $B_{p q}^{s} \|$. On calcule pour $t \leq 1$

$$
\begin{aligned}
\|t A U(t) u\|_{p} \leq & \left\|t A U(t) u_{-}(t)\right\|_{p}+\left\|t A U(t) u_{+}(t)\right\|_{p} \\
\leq & \left\|t A(1+A) U(t)(1+A)^{-1} u_{-}(t)\right\|_{p} \\
& +\left\|t A(1+A)^{-1} U(t)(1+A) u_{+}(t)\right\|_{p} \\
\leq & C t^{-1}\left\|u_{-}(t)\left|W_{p}^{-2}\|+t\| u_{+}(t)\right| W_{p}^{2}\right\|,
\end{aligned}
$$


parce que $t A U(t)$ et $t^{2} A^{2} U(t)$ sont bornés sur $X_{p}$ (et $t$ est borné), de même que $A(1+A)^{-1}$ et $U(t)$. En multipliant par $t^{-s / 2}$, on voit que

$$
\begin{aligned}
& \left\|t^{-s / 2}\right\| t A U(t) u\left\|_{p}\right\|_{q * 0} \\
& \quad \leq C\left(\left\|t^{s_{-}}\right\| u_{-}(t)\left|W_{p}^{-2}\|\|_{q *}+\left\|t^{s_{+}}\right\| u_{+}(t)\right| W_{p}^{2}\|\|_{q *}\right)
\end{aligned}
$$

en choisissant $s_{-}=-1-s / 2, s_{+}=1-s / 2$, qui vérifient les conditions (1.13) d'après la liaison entre $s$ et $\theta$ et $-2<s<2$. En passant à la borne inférieure, ceci montre bien que $\|u\|_{X} \leq C\left\|u \mid B_{p q}^{s}\right\|$.

Montrons ensuite $X \hookrightarrow B_{p q}^{s}(A)$. Puisque $X \subset W_{p}^{-2}(A)$, on peut écrire, dans cet espace, en vertu des propriétés analytiques du semigroupe,

$$
\begin{aligned}
u & =\int_{0}^{2} \tau A^{2} U(\tau) u d \tau+(1+2 A) U(2) u \\
& =\int_{0}^{2} \tau^{2} A^{2} U(\tau) u \frac{d \tau}{\tau}+c_{a} \int_{2}^{\infty} \tau^{-a}(1+2 A) U(2) u \frac{d \tau}{\tau}
\end{aligned}
$$

pour n'importe quel réel $a$ positif. Ainsi donc on a défini une fonction $u(\tau)$ telle que dans $W_{p}^{-2}(A)$ l'intégrale $\int_{0}^{\infty} u(\tau) / \tau d \tau$ converge, et vaut $u$. Pour $\tau \geq 2$, on majore, grâce à l'effet régularisant de $U(1)$,

$$
\begin{gathered}
\left\|u(\tau) \mid W_{p}^{-2}\right\| \leq \tau^{-a}\|U(1) u\|_{p}, \\
\left\|u(\tau) \mid W_{p}^{2}\right\| \leq \tau^{-a}\|U(1) u\|_{p} .
\end{gathered}
$$

Pour $\tau \leq 2$, on calcule

$$
\begin{gathered}
\left\|u(\tau) \mid W_{p}^{-2}\right\|=\left\|\tau^{2} A^{2}(1+A)^{-1} U(\tau) u\right\|_{p} \leq C \tau\left\|\frac{\tau}{2} A U\left(\frac{\tau}{2}\right) u\right\|_{p} \\
\left\|u(\tau) \mid W_{p}^{2}\right\|=\left\|\tau^{2} A^{2}(1+A) U(\tau) u\right\|_{p} \leq C \tau^{-1}\left\|\frac{\tau}{2} A U\left(\frac{\tau}{2}\right) u\right\|_{p}
\end{gathered}
$$

parce que $2 A(1+A)^{-1} U(\tau / 2)$ est borné sur $X_{p}$, de même que $\tau^{2} A^{2} U(\tau / 2)$ et $\tau^{3} A^{3} U(\tau / 2)$ (et $\tau$ est borné). Prenant par exemple $a=2$, on en déduit que

$$
\left\|\tau^{s_{-}}\right\| u(\tau)\left|W_{p}^{-2}\|\|_{q *}+\left\|\tau^{s_{+}}\right\| u(\tau)\right| W_{p}^{2}\|\|_{q *} \leq C\|u\|_{X}
$$

où $s_{-}$et $s_{+}$sont encore $-1-s / 2$ et $1-s / 2$, donc respectivement dans $]-2,0[$ et $] 0,2\left[\right.$. On a utilisé $s_{+}-a\left(\right.$ et $\left.s_{-}-a\right)$ négatif pour la 
convergence des intégrales en $\tau \rightarrow \infty$. Comme les conditions (1.13) sont vérifiées, ceci montre bien que $\left\|u \mid B_{p q}^{s}\right\| \leq C\|u\|_{X}$ (voir Proposition 48).

Remarque 15. Rappelons la définition des espaces de Besov usuels sur $\mathbb{R}^{n}$ au moyen d'une décomposition dyadique spectrale (voir par exemple [30]). On se donne $\chi \in C_{c}^{\infty}\left(\mathbb{R}^{n}\right)$, nulle hors de la boule de rayon 2 et égale à 1 sur la boule de rayon 1 . On pose $\varphi(\xi)=\chi\left(2^{-1} \xi\right)-\chi(\xi)$. Puis on note $\varphi_{-1}=\chi$ et, pour $j \in \mathbb{N}, \chi_{j}(\xi)=\chi\left(2^{-j} \xi\right)$ et $\varphi_{j}(\xi)=\varphi\left(2^{-j} \xi\right)$, de sorte que pour tout $k \in \mathbb{N}$

$$
1=\sum_{j \geq-1} \varphi_{j}(\xi)=\chi_{k}(\xi)+\sum_{j \geq k} \varphi_{j}(\xi), \quad \chi_{j}(\xi)=\sum_{k=-1}^{j-1} \varphi_{k}(\xi)
$$

On définit ensuite les opérateurs dyadiques $\Delta_{j}$ et $S_{j}$ par

$$
\widehat{\Delta_{j} u}(\xi)=\varphi_{j}(\xi) \widehat{u}(\xi), \quad \widehat{S_{j} u}(\xi)=\chi_{j}(\xi) \widehat{u}(\xi),
$$

où $\widehat{u}$ désigne la transformée de Fourier de $u$.

L'espace de Besov $B_{p q}^{s}\left(\mathbb{R}^{n}\right)$ est alors l'ensemble des distributions tempérées $u$ telles que

$$
\left\|S_{0} u\right\|_{p}+\left(\sum_{j=0}^{\infty} 2^{s j q}\left\|\Delta_{j} u\right\|_{p}^{q}\right)^{1 / q}<\infty
$$

La comparaison de (1.11) avec cette définition des espaces de Besov sur $\mathbb{R}^{n}$ révèle que $U(1)$ joue le rôle du filtre basse fréquence $S_{0}$, et que l'échelle $2^{j}$ (avec $j \geq 0$ ) et le filtre $\Delta_{j}$ associé à cette fréquence correspondent respectivement à la quantité $t^{-1 / 2}$ (avec $t \leq 1$ ) et à l' opérateur $t A U(t)$.

Vue leur définition, ces normes peuvent jouer un rôle important pour traiter de l'existence de solutions locales en temps. Le choix du temps 1 est arbitraire.

\subsubsection{Espaces de Besov "homogènes".}

Nous nous intéresserons au problème de l'existence globale. Il paraît alors judicieux d'introduire des normes qui prennent en compte 
les temps $t \longrightarrow \infty$. Soit $u$ dans l'un des espaces $W_{p}^{-2}(A)$. On sait faire opérer $U(t)$ sur $u$, et donc exprimer une condition portant sur $U(t) u$.

Définition 16. On note $\mathcal{A} \doteq \bigcup_{p \in] 1, \infty[} W_{p}^{-2}(A)$, et $I(2, p) \doteq$ ]$-2, \min \{2, n / p\}[$.

Pour $s \in I(2, p)$, on note $\dot{B}_{p q}^{s}(A)$ l'ensemble des $u \in \mathcal{A}$ tels que

$$
\left\|u \mid \dot{B}_{p q}^{s}\right\| \doteq\left\|t^{-s / 2}\right\| t A U(t) u\left\|_{p}\right\|_{q *}<\infty .
$$

Remarque 17. Pour les espaces de Besov homogènes définis sur $\mathbb{R}^{n}$ à partir d'une décomposition spectrale dyadique, on utilise alors $\dot{\Delta}_{j}$ pour $j \in \mathbb{Z}$.

Avec cette définition, il n'est pas évident que $\dot{B}_{p q}^{s}(A)$ soit un espace vectoriel, car $\mathcal{A}$ n'est pas stable par addition.

Proposition 18. $\dot{B}_{p q}^{s}(A)$ muni de $\left\|\cdot \mid \dot{B}_{p q}^{s}\right\|$ est un espace de Banach pour $s \in I(2, p)$.

$$
\dot{B}_{p_{1} q}^{s_{1}}(A) \hookrightarrow \dot{B}_{p_{2} q}^{s_{2}}(A) \text { pour } s_{i} \in I\left(2, p_{i}\right), p_{1} \leq p_{2} \text { et } s_{1}-n / p_{1}=
$$
$s_{2}-n / p_{2}$.

$$
\begin{aligned}
& \left.\dot{B}_{p q}^{s}(A) \hookrightarrow B_{p q}^{s}(A) \text { pour } s \in\right]-2,0[ \\
& \left.B_{p q}^{s}(A) \hookrightarrow \dot{B}_{p q}^{s}(A) \text { pour } s \in\right] 0, \min \{2, n / p\}[.
\end{aligned}
$$

DÉmonstration. Nous affirmons tout d'abord que pour $s \in]-2,0[$, $\dot{B}_{p q}^{s}(A)$ est en fait inclus dans $W_{p}^{-2}(A)$. En effet, si $u \in \dot{B}_{p q}^{s}(A)$, il existe $r$ tel que $u \in W_{r}^{-2}(A)$, et on peut donc écrire en vertu des propriétés analytiques du semi-groupe

$$
\begin{aligned}
u & =\int_{0}^{2} \tau A^{2} U(\tau) u d \tau+(1+2 A) U(2) u \\
& =\int_{0}^{2} \tau^{2} A^{2} U(\tau) u \frac{d \tau}{\tau}+(1+2 A) \int_{0}^{\infty} \tau A U(\tau) u \frac{d \tau}{\tau}
\end{aligned}
$$

On estime la norme de chacun des deux termes dans $W_{p}^{-2}(A)$

$$
\begin{aligned}
\|(1+2 A) \int_{2}^{\infty} \tau A U(\tau) & u \frac{d \tau}{\tau} \mid W_{p}^{-2} \| \\
\leq & \left\|\int_{2}^{\infty} \tau A U(\tau) u \frac{d \tau}{\tau}\right\|_{p}
\end{aligned}
$$




$$
\begin{gathered}
\leq \int_{2}^{\infty} \tau^{s / 2}\left(\tau^{-s / 2}\|\tau A U(\tau) u\|_{p}\right) \frac{d \tau}{\tau} \\
\leq\left\|\mathbf{1}_{[2, \infty[} \tau^{s / 2}\right\|_{q^{\prime} *}\left\|u \mid \dot{B}_{p q}^{s}\right\|, \\
\left\|\int_{0}^{2} \tau^{2} A^{2} U(\tau) u \frac{d \tau}{\tau} \mid W_{p}^{-2}\right\| \\
\left.\leq \int_{0}^{2} \tau^{2}\left\|(1+A)^{-1} A^{2} U(\tau) u\right\|_{p}\right) \frac{d \tau}{\tau} \\
\leq \int_{0}^{2} C \tau^{1+s / 2}\left(\left(\frac{\tau}{2}\right)^{-s / 2}\left\|\frac{\tau}{2} A U\left(\frac{\tau}{2}\right) u\right\|\right) \frac{d \tau}{\tau} \\
\leq C\left\|\mathbf{1}_{] 0,2[} \tau^{1+s / 2}\right\|_{q^{\prime} *}\left\|u \mid \dot{B}_{p q}^{s}\right\|
\end{gathered}
$$

où l'on a utilisé que $A(1+A)^{-1} U(\tau / 2)$ est borné sur $L^{p}$ uniformément en $\tau$. Les intégrales convergent précisément parce qu'on a supposé $s<0$ et $-2<s$.

Ainsi, quand $s \in]-2,0\left[\right.$, puisque $\dot{B}_{p q}^{s}(A) \subset W_{p}^{-2}(A)$, on en déduit que $\dot{B}_{p q}^{s}(A)$ est un espace vectoriel et que $\left\|u \mid \dot{B}_{p q}^{s}\right\|$ est une norme. L'inclusion correspond à une injection continue. De plus, on vient de voir que $\|U(2) u\|_{p} \leq C\left\|u \mid \dot{B}_{p q}^{s}\right\|$. Le même calcul montre que cette majoration vaut aussi pour $U(1) u$. On en déduit $\left\|u\left|B_{p q}^{s}\|\leq C\| u\right|\right.$ $\dot{B}_{p q}^{s} \|$ et $\dot{B}_{p q}^{s}(A) \hookrightarrow B_{p q}^{s}(A)$.

Soient $s_{i} \in I\left(2, p_{i}\right), i=1,2$. D'après les inégalité $L^{p_{1}}-L^{p_{2}}$ sur le semi-groupe, pour $p_{1} \leq p_{2}$,

$$
\begin{aligned}
t^{-s_{2} / 2}\|t A U(t) u\|_{p_{2}} & =t^{-s_{2} / 2} 2\left\|U\left(\frac{t}{2}\right) \frac{t}{2} A U\left(\frac{t}{2}\right) u\right\|_{p_{2}} \\
& \leq C t^{-s_{2} / 2-\left[p_{1}, p_{2}\right]}\left\|\frac{t}{2} A U\left(\frac{t}{2}\right) u\right\|_{p_{1}} .
\end{aligned}
$$

Et comme $-s_{2} / 2-\left[p_{1}, p_{2}\right]=-\left(s_{2}-n / p_{2}+n / p_{1}\right) / 2$, on obtient en prenant la norme dans $L^{q *}$

$$
\left\|u\left|\dot{B}_{p_{2} q}^{s_{2}}\|\leq C\| u\right| \dot{B}_{p_{1} q}^{s_{1}}\right\|, \quad \text { pour } s_{2}-\frac{n}{p_{2}}=s_{1}-\frac{n}{p_{1}} .
$$

Cela implique $\dot{B}_{p_{1} q}^{s_{1}}(A) \subset \dot{B}_{p_{2} q}^{s_{2}}(A)$. Vu que $s_{1}-n / p_{1}<0$, on peut toujours trouver $\left.s_{2} \in\right]-2,0\left[\right.$ et $p_{2} \in\left[p_{1}, \infty\left[\right.\right.$ vérifiant $s_{2}-n / p_{2}=$ 
$s_{1}-n / p_{1}$. Alors $\dot{B}_{p_{1} q}^{s_{1}}(A) \subset W_{p_{2}}^{-2}(A)$; comme précédemment cela entraîne que $\dot{B}_{p_{1} q}^{s_{1}}(A)$ est un espace vectoriel normé et que les inégalités correspondent à des injections continues. À ce stade, on vérifie sans peine que les espaces $\dot{B}_{p q}^{s}(A)$ pour $s \in I(2, p)$ sont complets.

Enfin, soit $0<s<\min \{2, n / p\}$ et $u \in B_{p q}^{s}(A)$. Par (1.9) et le Corollaire 4,

$$
t^{1-s / 2}\|A U(t) u\|_{p} \leq C t^{-s / 2}\|u\|_{p} \in L^{q * \infty}
$$

car $s>0$. Donc $u \in \dot{B}_{p q}^{s}(A)$.

Ajoutons deux résultats qui renforcent le lien avec le semi-groupe.

Lemme 19. Soit $s<0$. La quantité $\left\|t^{-s / 2}\right\| U(t) u\left\|_{p}\right\|_{q *}$ définit sur $\dot{B}_{p q}^{s}(A)$ une norme équivalente.

DÉmonstration. De la majoration $\|t A U(t) u\|_{p} \leq C\|U(t / 2) u\|_{p}$ uniforme par rapport à $t$ on tire directement

$$
\left\|u \mid \dot{B}_{p q}^{s}\right\| \leq C\left\|t^{-s / 2}\right\| U(t) u\left\|_{p}\right\|_{q_{*}} .
$$

Inversement, si $u \in \dot{B}_{p q}^{s}(A)$, comme $s<0$ on sait que $u \in W_{p}^{-2}(A)$ et on peut écrire

$$
U(t) u=\int_{t}^{\infty} \tau A U(\tau) u \frac{d \tau}{\tau}
$$

Donc

$$
\begin{aligned}
t^{-s / 2}\|U(t) u\|_{p} & \leq \int_{t}^{\infty}\left(\frac{t}{\tau}\right)^{-s / 2}\left(\tau^{-s / 2}\|\tau A U(\tau) u\|_{p}\right) \frac{d \tau}{\tau} \\
& \leq\left(\mathbf{1}_{] 0,1]} \tau^{-s / 2}\right) *\left(\tau^{-s / 2}\|\tau A U(\tau) u\|_{p}\right)
\end{aligned}
$$

où ici $*$ désigne la convolution sur le groupe $\left(\mathbb{R}_{+}, \times, d t / t\right)$. L'inégalité de Hölder-Young donne alors

$$
\begin{aligned}
\left\|t^{-s / 2}\right\| U(t) u\left\|_{p}\right\|_{q *} & \leq\left\|\mathbf{1}_{] 0,1]} \tau^{-s / 2}\right\|_{1 *}\left\|\tau^{-s / 2}\right\| \tau A U(\tau) u\left\|_{p}\right\|_{q *} \\
& \leq C\left\|u \mid \dot{B}_{p q}^{s}\right\|
\end{aligned}
$$

puisque $s<0$. 
Remarque 20. Dans le cas de $\mathbb{R}^{3}$, en prenant $A=-\Delta$ et donc $U(t)=$ $\exp (t \Delta)$, ce lemme correspond à [4, Lemme 3.3.3]. C'est un point crucial dans la relecture par [4] du résultat de [16].

Lemme 21. Soit $s \in I(2, p)$ et $q \in[1, \infty]$. L'application $t \longmapsto U(t)$ est continue bornée de $\mathbb{R}_{+}$dans $\mathcal{L}\left(\dot{B}_{p q}^{s}(A)\right)$. Si de plus $s-n / p+n / r \in$ ]$-2,0\left[\right.$ et $p \leq r$ alors $\lim _{t \rightarrow 0} U(t) u=u$ dans $W_{r}^{-2}(A)$ pour tout $u \in$ $\dot{B}_{p q}^{s}(A)$.

DÉmonstration. La continuité en 0 dans $W_{r}^{-2}(A)$ vient des injections

$$
\dot{B}_{p q}^{s}(A) \hookrightarrow \dot{B}_{r q}^{\varsigma}(A) \hookrightarrow B_{r q}^{\varsigma}(A) \hookrightarrow W_{r}^{-2}(A), \quad \varsigma=s-\frac{n}{p}+\frac{n}{r}
$$

et de la continuité de $U(t)$ dans cet espace.

La borne et la continuité de $U(t)$ dans $\mathcal{L}\left(\dot{B}_{p q}^{s}(A)\right)$ découlent des même propriétés dans $\mathcal{L}\left(X_{p}\right)$, par commutation de $U(t)$ et $\theta^{1-s / 2} A U(\theta)$.

\section{2. Énoncés.}

On considère l'équation de Navier-Stokes dans $\Omega$ pour la viscosité $\varepsilon=1$

$$
\begin{cases}\partial_{t} u-\Delta u+\nabla \cdot(u \otimes u)+\nabla p=0, & \\ \nabla \cdot u=0, & \gamma_{0} u=u_{\mid \partial \Omega}=0,\end{cases}
$$

avec la donnée initiale $u_{\mid t=0}=u_{0}$. On a écrit $\nabla \cdot(u \otimes u)$ pour

$$
\sum_{k} \partial_{k}\left(u_{k} u_{i}\right)
$$

En appliquant le projecteur $P$, on se ramène à

$$
\partial_{t} u+A u+P \nabla \cdot(u \otimes u)=0, \quad u_{\mid t=0}=u_{0}, \gamma_{0} u=0,
$$

dont on cherche les solutions sous la forme

$$
u(t)=U(t) u_{0}-\int_{0}^{t} U(t-\tau) P \nabla \cdot(u \otimes u)(\tau) d \tau .
$$


Considérant le second terme du membre de droite comme un opérateur (quadratique) appliqué à $u$, on lit (2.2) comme une équation de point fixe.

Définition 22. On note $U_{0} \doteq U(t) u_{0}$. On note $\Theta$ l'opérateur bilinéaire

$$
\Theta(u, v)(t, x) \doteq \int_{0}^{t} U(t-\tau) P \nabla \cdot(u \otimes v)(\tau, x) d \tau
$$

où $\nabla \cdot(u \otimes v)$ désigne le champ de vecteurs $\sum_{k} \partial_{k}\left(u^{k} v^{i}\right)$.

Ainsi on cherche à résoudre l'équation de point fixe

$$
u=U_{0}-\Theta(u, u) \text {. }
$$

Théorème 23. Il existe $\eta \in C(] n, \infty\left[; \mathbb{R}_{+}\right)$vérifiant ceci. Pour tout $u_{0} \in X_{n}$, s'il existe $p>n$ tel que $\left\|u_{0} \mid \dot{B}_{p \infty}^{-1+n / p}\right\|<\eta(p)$, alors il existe $u \in C_{b}\left(\overline{\mathbb{R}}_{+} ; X_{n}\right)$ solution de l'équation $(2.2)$ avec $u(0)=u_{0}$.

$u$ est l'unique solution de (2.2) parmi les fonctions $v$ de $C\left(\mathbb{R}_{+} ; X_{n}\right)$ vérifiant $\sup _{t>0} t^{(1-n / p) / 2}\|v(t)\|_{p}<2 \eta(p)$.

Pour le cas de $\mathbb{R}^{3}$, ce résultat est dû à $[4,28]$ L'intérêt de n'imposer la petitesse que sur la norme dans un espace de Besov se voit bien dans le lemme suivant, qui est une adaptation au cas de notre domaine extérieur d'un résultat semblable sur $\mathbb{R}^{3}$ de [14].

Lemme 24. Soit $n<p$. Il existe une suite $u^{k} \in X_{n}$ telle que

$$
1<\inf _{k}\left\|u^{k}\right\|_{n} \quad \text { et } \quad \lim _{k \rightarrow \infty}\left\|u^{k} \mid \dot{B}_{p \infty}^{-1+\frac{n}{p}}\right\|=0 .
$$

On peut étendre l'ensemble des données initiales de la façon suivante :

Théorème 25. Il existe $\eta \in C(] n, \infty\left[; \mathbb{R}_{+}\right)$vérifiant ceci. Pour tout $r \in] n, \infty\left[\right.$ et $u_{0} \in \dot{B}_{r \infty}^{-1+n / r}(A)$, s'il existe $p \geq r$ tel que $\left\|u_{0} \mid \dot{B}_{p \infty}^{-1+n / p}\right\|$ $<\eta(p)$, alors il existe $u \in C_{b}\left(\mathbb{R}_{+} ; \dot{B}_{r}^{-1+n / r}(A)\right)$ solution de l'équation $(2.2)$ avec $u(t) \longrightarrow u_{0}$ dans $W_{r}^{-2}(A)$ quand $t \longrightarrow 0$.

$u$ est l'unique solution de (2.2) parmi les fonctions $v$ de $C_{b}\left(R_{+} ; \dot{B}_{r \infty}^{-1+n / r}(A)\right)$ vérifiant $\sup _{t>0} t^{(1-n / p) / 2}\|v(t)\|_{p}<2 \eta(p)$. 
Comme dans le cas de $\mathbb{R}^{3}$, ces théorèmes d'existence, obtenus par un point fixe dans un espace de Banach plus petit que celui induit naturellement par la donnée initiale, ne sont pas satisfaisants pour leur assertion sur l'unicité. On y demande la condition supplémentaire $\sup _{t>0} t^{(1-n / p) / 2}\|v(t)\|_{p}<2 \eta(p)$. Ceci est relié au fait qu'on ne sait pas si l'opérateur bilinéaire $\Theta$ est continu dans $C_{b}\left(\mathbb{R}_{+}, X_{n}\right)$. D'après [26], il ne l'est pas dans $L^{3}\left(\mathbb{R}^{3}\right)$, tandis qu'il l'est dans $L^{3, \infty}\left(\mathbb{R}^{3}\right)$, d'après [24]. Cette difficulté est contournée dans [7], [8] par l'utilisation de normes différentes pour les deux arguments de l'opérateur bilinéaire. Nous avons adapté leur résultat au cas de $\Omega$ ouvert extérieur.

Théorème 26. Soit $u_{0} \in X_{n}$ et $U_{0} \doteq U(t) u_{0}$. Soit $u^{1}$ et $u^{2}$ dans $C_{b}\left(\left[0, T\left[; X_{n}\right)\right.\right.$, solutions de l'équation $(2.2)$. Alors $u^{1}=u^{2}$ sur $[0, T[$.

\section{Démonstrations des théorèmes.}

Nous rassemblons d'abord les résultats de continuité sur $\Theta$ utilisés ensuite. Puis nous prouvons les deux théorèmes d'existence. Enfin le théorème d'unicité.

\subsection{Continuité de l'opérateur bilinéaire $\Theta$.}

\subsubsection{Pour l'existence.}

Définition 27. On définit

$$
[p]=[n, p]=\frac{1}{2}\left(1-\frac{n}{p}\right)
$$

de sorte que $[p, q]=[q]-[p]$.

On définit à la manière de [27] l'espace de Banach $E_{p}$ comme l'ensemble des $u$ continus de $\mathbb{R}_{+}$dans $X_{p}$ tels que

$$
\|u\|_{E_{p}} \doteq \sup _{t>0}\left\|t^{[p]} u(t)\right\|_{p}<\infty
$$

Pour $T_{*}=0$ ou $\infty$, on définit aussi le sous-espace $($ fermé $) E_{p ; 0}\left(T_{*}\right)$ de $E_{p}$ par

$$
E_{p ; 0}\left(T_{*}\right)=\left\{u(t, x) \in E_{p}: \lim _{t \rightarrow T_{*}}\left\|t^{[p]} u(t)\right\|_{p}=0\right\} .
$$


Enfin on abrège $\dot{B}_{p \infty}^{-1+n / p}(A)$ en $\dot{\mathcal{B}}_{p}$.

Proposition 28. Étant donnés $p, q$ et $r$ dans $] 1, \infty[$ tels que

$$
\frac{1}{r} \leq \frac{1}{p}+\frac{1}{q} \leq \frac{1}{r}+\frac{1}{n}
$$

l'opérateur bilinéaire $\Theta$ est continu de $E_{p} \times E_{q}$ dans $C\left(\mathbb{R}_{+} ; W_{r}^{-2}(A)\right)$, et

$$
\left\|\Theta(u, v)(t) \mid W_{r}^{-2}\right\| \leq C t^{n(1 / p+1 / q) / 2}\|u\|_{E_{p}}\|v\|_{E_{q}} .
$$

En particulier, $\lim _{t \rightarrow 0} \Theta(u, v)(t)=0$ dans $W_{r}^{-2}(A)$.

Proposition 29. Étant donnés $p, q$ et $r$ dans $] 1, \infty[$ tels que

$$
\frac{1}{p}+\frac{1}{q}-\frac{1}{n}<\frac{1}{r} \leq \frac{1}{p}+\frac{1}{q} \leq \frac{1}{n^{\prime}}
$$

l'opérateur bilinéaire $\Theta$ est continu de $E_{p} \times E_{q}$ dans $E_{r}$.

De plus, pour $T_{*}=0$ ou $\infty$, si $u \in E_{p ; 0}\left(T_{*}\right)$ ou $v \in E_{q ; 0}\left(T_{*}\right)$ alors $\Theta(u, v) \in E_{r ; 0}\left(T_{*}\right)$.

Proposition 30. Étant donnés $p, q$ et $r$ dans $] 1, \infty[$ tels que

$$
\frac{1}{r} \leq \frac{1}{p}+\frac{1}{q}<\frac{2}{n}
$$

l'opérateur bilinéaire $\Theta$ est continu de $E_{p} \times E_{q}$ dans $C_{b}\left(\mathbb{R}_{+} ; \dot{\mathcal{B}}_{r}\right)$.

Notation 31. On note $s$ le nombre tel que $1 / p+1 / q=1 / s$, et $a \doteq$ $-[p]-[q]=-1+n /(2 s)$. Pour $(u, v) \in E_{p} \times E_{q}$, on note $\widetilde{u}(t) \doteq$ $t^{[p]} u(t)$ et $\widetilde{v}(t) \doteq t^{[q]} v(t)$, si bien que $\widetilde{u} \otimes \widetilde{v} \in C_{b}\left(\mathbb{R}_{+} ; L^{s}(\Omega)\right)$ avec $\sup _{t}\|(\widetilde{u} \otimes \widetilde{v})(t)\|_{s} \leq\|u\|_{E_{p}}\|v\|_{E_{q}}$ et $(u \otimes v)(t)=t^{a}(\widetilde{u} \otimes \widetilde{v})(t)$.

On note $\widetilde{U}(t) \doteq t^{-b} U(t) P \nabla \cdot$, où l'exposant $b$ s'adapte à chaque proposition, et $\widetilde{w}(t, \tau) \doteq \widetilde{U}(t(1-\tau))(\widetilde{u} \otimes \widetilde{v})(t \tau)$.

Enfin on note

$$
f(t, \tau) \doteq t U(t(1-\tau)) P \nabla \cdot(u \otimes v)(t \tau)
$$

de sorte que

$$
\Theta(u, v)(t)=\int_{0}^{1} f(t, \tau) d \tau
$$


et

$$
f(t, \tau)=t^{1+a+b} \tau^{a}(1-\tau)^{b} \widetilde{w}(t, \tau)
$$

Preuve de la Proposition 28. D'après la Proposition 6, pour $s, r$ dans ]1, $\infty[$ tels que $0 \leq 1 / s-1 / r \leq 1 / n$ et $b=0, \widetilde{U}(t) \in$ $C_{b}\left(\mathbb{R}_{+} ; \mathcal{L}\left(L^{s}(\Omega) ; W_{r}^{-2}(A)\right)\right)$. Alors $\widetilde{w} \in C_{b}\left(\mathbb{R}_{+} \times\right] 0,1\left[; W_{r}^{-2}(A)\right)$, et comme $a>-1$, le résultat découle du théorème de continuité sous le signe $\int$ appliquée à $\int_{0}^{1} f(t, \tau) d \tau$.

Preuve de la Proposition 29. D'après le Corollaire 10, pour $s, r$ dans $] 1, \infty\left[\right.$ tels que $1 / r \leq 1 / s \leq 1 / n^{\prime}$ et $b=-1 / 2-[s, r], \widetilde{U}(t) \in$ $C_{b}\left(\mathbb{R}_{+} ; \mathcal{L}\left(L^{s}(\Omega) ; X_{r}\right)\right)$. Alors $\widetilde{w} \in C_{b}\left(\mathbb{R}_{+} \times\right] 0,1\left[; X_{r}\right)$. Comme $[r]+1+$ $a+b=0$ et $a>-1$, on obtient le résultat par le théorème de continuité sous le signe $\int$ pourvu que $b>-1$, ce qui s'écrit encore $1 / s-1 / n<1 / r$.

Si $\lim _{t \rightarrow T_{*}} \widetilde{u}=0$ dans $X_{p}$ (respectivement $\widetilde{v}, X_{q}$ ), alors

$$
\lim _{t \rightarrow T_{*}} \widetilde{w}(t, \tau)=0
$$

dans $X_{r}$ uniformément en $\tau$ sur tout compact de $] 0,1[$. On en déduit facilement que $\lim _{t \rightarrow T_{*}} t^{[r]} \Theta(\widetilde{u}, \widetilde{v})=0$ dans $X_{r}$.

Preuve de la Proposition 30. On va montrer que, pour $b=$ $-n /(2 s)$ et $r, s$ dans $] 1, \infty\left[\right.$ tels que $1 / r \leq 1 / s \leq 1 / n^{\prime}$ et $1 / r \leq 3 / n$, $\widetilde{U}(t) \in C_{b}\left(\mathbb{R}_{+} ; \mathcal{L}\left(L^{s}(\Omega) ; \dot{\mathcal{B}}_{r}\right)\right)$. Alors $\widetilde{w} \in C_{b}\left(\mathbb{R}_{+} \times\right] 0,1\left[; \dot{\mathcal{B}}_{r}\right)$. Comme $1+a+b=0$ et $a>-1$, on obtient la proposition par le théorème de continuité sous le signe $\int$ pourvu que $b>-1$, ce qui s'écrit encore $1 / s<2 / n$.

Commençons par la borne. Soit $c \doteq 1+[r]$ et $w \in L^{s}(\Omega)$. Par définition

$$
\sup _{t>0}\left\|\widetilde{U}(t) w \mid \dot{\mathcal{B}}_{r}\right\|=\sup _{t, \theta>0} \theta^{c} t^{-b}\|A U(t+\theta) P \nabla \cdot w\|_{r}
$$

D'après le Corollaire 10, $\|A U(t+\theta) P \nabla \cdot w\|_{r} \leq C(t+\theta)^{b-c}\|w\|_{s}$ pour $n^{\prime} \leq s \leq r$, car $1+1 / 2+[s, r]=c-b$. Or $\theta^{c} t^{-b}(t+\theta)^{b-c} \leq 1$ pour $b \leq 0 \leq c$ et $(t, \theta) \in \mathbb{R}_{+} \times \mathbb{R}_{+}$. On en déduit la borne dès que $n / r \leq 3$.

Finissons par la continuité, qui découle de

$$
\widetilde{U}(t+h)-\widetilde{U}(t)=\left(U\left(\frac{t}{2}+h\right)-U\left(\frac{t}{2}\right)\right) \widetilde{U}\left(\frac{t}{2}\right)
$$


et de $U(t) \in C_{b}\left(\mathbb{R}_{+} ; \mathcal{L}\left(\dot{\mathcal{B}}_{r}\right)\right)$ vu au Lemme 21 .

\subsubsection{Pour l'unicité.}

Définition 32. On pose $\bar{\Theta}(u, v) \doteq \Theta(v, u)$. On note $L_{T}^{\infty}(X)$ les fonctions fortement mesurables bornées de ]0,T[ dans un espace de Banach $X$. On définit l'espace de Banach $F_{p}^{T}$ comme l'ensemble des u mesurables de $] 0, T\left[\right.$ dans $X_{p}$ tels que

$$
\|u\|_{F_{p}^{T}} \doteq \sup _{0<t<T}\left\|t^{[p]} u(t)\right\|_{p}<\infty .
$$

On abrège $B_{p \infty}^{-n / q+n / p}(A)$ en $\mathcal{B}_{p}^{q}$, et $\mathcal{B}_{p}^{n}$ en $\mathcal{B}_{p}$. Enfin $G_{p}^{T} \doteq L_{T}^{\infty}\left(\mathcal{B}_{p}\right)$.

Proposition 33. Étant donnés $p, q$ et $r$ dans $] 1, \infty[$ tels que

$$
\frac{1}{r}-\frac{1}{q} \leq \frac{1}{p} \leq \frac{1}{n}
$$

il existe $C$ tel que pour tout $T \in] 0,1[$, les opérateurs bilinéaires $\Theta$ et $\bar{\Theta}$ sont continus de $F_{p}^{T} \times L_{T}^{\infty}\left(X_{q}\right)$ dans $L_{T}^{\infty}\left(\mathcal{B}_{r}^{q}\right)$ avec une norme majorée par $C$.

Démonstration. Posons $c=1+[q, r]$. Par définition de $\| \Theta(u, v)(t) \mid$ $\mathcal{B}_{r}^{q} \|$, on cherche $C$ tel que pour $u \in F_{p}^{T}, v \in L_{T}^{\infty}\left(X_{q}\right)$ et $0<t<T<1$,

$$
\begin{aligned}
\|U(1) \Theta(u, v)(t)\|_{r}+\sup _{0<\theta<1} \| \theta^{c} A U(\theta) & \Theta(u, v)(t) \|_{r} \\
& \leq C\|u\|_{F_{p}^{T}}\|v\|_{L_{T}^{\infty}\left(X_{q}\right)} .
\end{aligned}
$$

Or

$$
\|(u \otimes v)(\tau)\|_{s} \leq \tau^{a}\|u\|_{F_{p}^{T}}\|v\|_{L_{T}^{\infty}\left(X_{q}\right)}
$$

avec $1 / s=1 / p+1 / q$ et $a=-[p]$.

Et

$$
U(1) \Theta(u, v)(t)=\int_{0}^{t} U(1+t-\tau) P \nabla \cdot(u \otimes v)(\tau) d \tau .
$$

D'après le Corollaire 10, $U(\vartheta) P \nabla$. est borné dans $\mathcal{L}\left(L^{s}(\Omega) ; X_{r}\right)$ pour $\vartheta=1+t-\tau \in] 1,2\left[\right.$ et $s \leq r$. Comme $\int_{0}^{t} \tau^{a} d \tau \leq 2$, on obtient la première partie de (3.6). 
Et

$$
\theta^{c} A U(\theta) \Theta(u, v)(t)=\int_{0}^{t} \theta^{c} A U(\theta+t-\tau) P \nabla \cdot(u \otimes v)(\tau) d \tau .
$$

D'après le Corollaire $10, \vartheta^{-b} U(\vartheta) P \nabla$. est borné dans $\mathcal{L}\left(L^{s}(\Omega) ; X_{r}\right)$ avec $b=-3 / 2-[s, r]$, pour $\vartheta=\theta+t-\tau \in] 0,2[$ et $s \leq r$. Donc

$$
\begin{aligned}
& \| \theta^{c} A U(\theta) \Theta(u, v)(t) \|_{r} \\
& \leq C\|u\|_{F_{p}^{T}}\|v\|_{L_{T}^{\infty}\left(X_{q}\right)} \int_{0}^{t} \theta^{c}(\theta+t-\tau)^{b} \tau^{a} d \tau .
\end{aligned}
$$

L'intégrale devient

$$
I=\int_{0}^{1} \xi^{c}(\xi+1-\tau)^{b} \tau^{a} d \tau
$$

si on pose $\theta=t \xi$, car $a+b+c=-1$. Si $-1<a<0 \leq c$, alors

$$
I \leq \int_{0}^{1}(1-\tau)^{-1-a} \tau^{a} d \tau
$$

car alors, pour $(\xi, \tau) \in \mathbb{R}_{+} \times \mathbb{R}_{+}, \xi^{c}(\xi+1-\tau)^{b}(1-\tau)^{1+a}<1$. Si $a=0<c$, alors $I<1 / c$. Or (3.5) implique $a \in]-1 / 2,0]$ et $1 / 2 \leq c$. On obtient donc la seconde partie de (3.6).

Corollaire 34. Étant donnés $p, q$ et $r$ dans $] 0,1[$ tel que

$$
0<\frac{1}{r}-\frac{1}{n}<\frac{1}{p} \leq \frac{1}{n}
$$

il existe $C$ tel que pour tout $T \in[0,1]$, les opérateurs bilinéaires $\Theta$ et $\bar{\Theta}$ sont continus de $F_{p}^{T} \times G_{r}^{T}$ dans $G_{r}^{T}$ avec une norme majorée par $C$.

DÉmonstration. D'une part $L^{n \infty}(\Omega)=\left(L^{q}(\Omega), L^{o}(\Omega)\right)_{\theta, \infty}$ dès que

$$
\frac{1}{n}=\frac{1-\theta}{q}+\frac{\theta}{o} .
$$

Quand on se restreint aux champs à divergence nulle et tangents au bord, on obtient $X_{n \infty}=\left(X_{q}, X_{o}\right)_{\theta, \infty}$ (voir [19]). D'autre part, $\mathcal{B}_{r}=$ $\left(\mathcal{B}_{r}^{q}, \mathcal{B}_{r}^{o}\right)_{\theta, \infty}$ d'après le théorème de réitération. Par ailleurs, pour $r<n$, 
$B_{r \infty}^{-1+n / r}(\Omega) \hookrightarrow L^{n \infty}(\Omega)$ par injection de Sobolev et interpolation réelle (voir [30]), et $\mathcal{B}_{r} \hookrightarrow X_{r} \cap B_{r \infty}^{-1+n / r}(\Omega)$ d'après [17, Lemma 4.4]. On en déduit $\mathcal{B}_{r} \hookrightarrow X_{n \infty}$. Enfin, la théorie de l'interpolation (voir par exemple [20]) nous enseigne que, pour tout $\theta \in] 0,1\left[, L_{T}^{\infty}\left(\left(A_{0}, A_{1}\right)_{\theta, \infty}\right)=\right.$ $\left(L_{T}^{\infty}\left(A_{0}\right), L_{T}^{\infty}\left(A_{1}\right)\right)_{\theta, \infty}$. Donc

$$
\left(L_{T}^{\infty}\left(\mathcal{B}_{r}^{q}\right), L_{T}^{\infty}\left(\mathcal{B}_{r}^{o}\right)\right)_{\theta, \infty}=G_{r}^{T} \hookrightarrow L_{T}^{\infty}\left(X_{n \infty}\right)=\left(L_{T}^{\infty}\left(X_{q}\right), L_{T}^{\infty}\left(X_{o}\right)\right)_{\theta, \infty} .
$$

On fixe maintenant $u \in F_{p}^{T}$, et on considère les opérateurs linéaires $v \longmapsto \Theta(u, v)$ et $v \longmapsto \bar{\Theta}(u, v)$. On leur applique la Proposition 33 et la propriété fondamentale de l'interpolation (voir Théorème 49). Il reste à choisir $o \leq q$ vérifiants (3.9) et (3.5), ce qui est possible d'après (3.8) en prenant $q$ assez proche de $n$.

\subsection{Existence globale à donnée petite.}

\subsubsection{Point fixes.}

Lemme 35. Soit $E$ un espace de Banach, $U_{0} \in E$ et $\Theta: E \times E \longrightarrow E$ une application bilinéaire avec $\|\Theta(u, v)\|_{E} \leq C\|u\|_{E}\|v\|_{E}$. On note $f: E \longrightarrow E$ l'application continue $f(u)=U_{0}-\Theta(u, u)$.

1) Si $\left\|U_{0}\right\|_{E}<(4 C)^{-1}$, alors l'équation de point fixe $f(u)=u$ admet une solution dans la boule fermée de rayon

$$
(2 C)^{-1}\left(1-\sqrt{1-4 C\left\|U_{0}\right\|_{E}}\right) .
$$

2) L'équation de point fixe $f(u)=u$ admet au plus une solution dans la boule ouverte de rayon $(2 C)^{-1}$.

La preuve est élémentaire.

Voici maintenant la partie existence globale à donnée petite. Rappelons que $u_{0} \in \mathcal{A}$ s'il existe $\left.p \in\right] 1, \infty\left[\right.$ tel que $u_{0} \in W_{p}^{-2}(A)$. Dans ce cas, on sait faire opérer $U(t)$ sur $u_{0}$, et donc exprimer une hypothèse du genre $U(t) u_{0} \in E_{p}$. Comme le point fixe fait intervenir $U_{0}$ plutôt que $u_{0}$, on énonce le résultat en ces termes :

Proposition 36 (Existence). Soit $n<p$, et $u_{0} \in \mathcal{A}$ tel que $U_{0} \doteq$ $U(t) u_{0} \in E_{p}$ avec

$$
\left\|U_{0}\right\|_{E_{p}}<(4 C)^{-1}
$$


où $C$ est la norme de l'opérateur bilinéaire $\Theta: E_{p} \times E_{p} \longrightarrow E_{p}$. Alors il existe une solution $u \in E_{p}$ à l'équation (2.2) qui est unique dans la boule de cet espace de rayon $(2 C)^{-1}$. De plus $\|u\|_{E_{p}} \leq 2\left\|U_{0}\right\|_{E_{p}}$.

Pour $T_{*}=0$ ou $\infty$, si $U_{0} \in E_{p ; 0}\left(T_{*}\right)$ alors $u \in E_{p ; 0}\left(T_{*}\right)$.

La preuve est une application immédiate du lemme précédent et de la continuité de l'opérateur bilinéaire énoncé dans la Proposition 29. Ajoutons seulement que $p>n \geq 3$ implique $2 / p<2 / n \leq 1 / n^{\prime}$. Et que pour $x \leq(4 C)^{-1},(2 C)^{-1}(1-\sqrt{1-4 C x}) \leq 2 x$.

Remarque 37. Le Lemme 39 ci-dessous permettra de transférer l'hypothèse de petitesse de $\left\|U_{0}\right\|_{E_{p}}$ à $\left\|u_{0} \mid \dot{\mathcal{B}}_{p}\right\|$. Mais il n'est pas complètement vain de garder à l'esprit que, plus que $u_{0}$, c'est $U_{0}$ qui est réellement la donnée du problème. Si on étudiait par exemple l'équation de Navier-Stokes avec un terme de force (un $f$ au second membre de (2.1)), on s'arrangerait pour le faire rentrer dans $U_{0}$. Il resterait ensuite à trouver des conditions sur $f$ suffisantes pour que le $U_{0}$ ainsi déterminé vérifie les hypothèses du théorème.

On va maintenant exploiter plus largement la Proposition 29 pour obtenir des renseignements supplémentaires sur la solution. Il s'agit de résultats de régularité, qui rappellent ceux de l'équation de la chaleur.

Proposition 38 (Régularité). Soit $n<p$, et $u_{0} \in \mathcal{A}$ tel que $U_{0} \doteq$ $U(t) u_{0} \in E_{p}$. Alors $U_{0} \in E_{s}$ pour $p \leq s$.

Si de plus $u \in E_{p}$ est une solution de l'équation (2.2) alors $u \in E_{s}$ pour $p \leq s$ et $u-U_{0} \in E_{s}$ pour $p / 2 \leq s$.

Si de plus $U_{0} \in E_{q}$ pour un $q \in\left[n, p\left[\right.\right.$, alors $u$ et $U_{0}$ sont dans $E_{s}$ pour $q \leq s$, et $u-U_{0} \in E_{s}$ pour $q / 2 \leq s$.

Pour $T_{*}=0$ ou $\infty$, tout ceci est encore valable quand on remplace les espaces $E_{\star}$ par leur variante $E_{\star ; 0}\left(T_{*}\right)$.

DÉmonstration. On remarque que $n \geq 3$ entraîne $2 / n \leq 1 / n^{\prime}$.

Tout d'abord, on constate que si $U_{0} \in E_{p}$, alors $U_{0} \in E_{s}$ pour $1 / s \in] 0,1 / p]$, par l'estimation $L^{p}-L^{s}$ du semi-groupe (1.6).

Ensuite, comme $n<p$, on vérifie $2 / p<2 / n \leq 1 / n^{\prime}$, et donc $u \in E_{p}$ implique d'après la Proposition 29 que $\Theta(u, u) \in E_{r}$ pour $1 / r \in] \max \{0,2 / p-1 / n\}, 2 / p]$, donc $u=U_{0}+\Theta(u, u) \in E_{s}$ pour $1 / s \in] \max \{0,2 / p-1 / n\}, 1 / p]$. On montre ensuite par récurrence sur $k$ que pour tout $k$ l'assertion : " $u \in E_{s}$ pour tout $s$ vérifiant $1 / s \in$ 
$\left.\max \left\{0,2^{k} / p-\left(2^{k}-1\right) / n\right\}, 1 / p\right] "$ est vraie. En effet pour $k=1$, on vient de le voir. Et pour passer de $k$ à $k+1$, on écrit encore $u=U_{0}+\Theta(u, u)$ et on applique à nouveau la Proposition 29. Voir sur la Figure 1 la flèche issue de $1 / p_{1}$. Chaque segment vertical de la flèche représente l'intervalle des valeurs de $1 / s$ qu'on ajoute à chaque itération. Le fait que $u-U_{0} \in E_{s}$ pour $1 / s \in[1 / p, 2 / p]$ découle de l'assertion déjà démontrée " $\Theta(u, u) \in E_{r}$ pour $\left.\left.1 / r \in\right] \max \{0,2 / p-1 / n\}, 2 / p\right]$ ". Les autres valeurs de $s$ s'obtiennent en considérant $u$ et $U_{0}$ séparément.

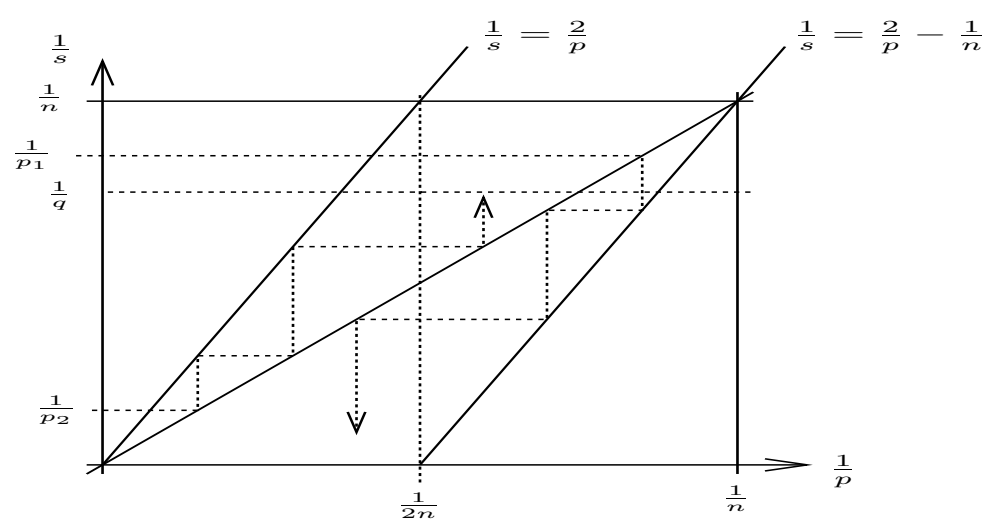

Figure 1. Propriétés supplémentaires.

On utilise le même argument pour les valeurs de $s$ plus petites que $p$, avec l'hypothèse que $U_{0} \in E_{q}$ et $n \leq q<p$. Si $U_{0} \in E_{q}$ alors, par l'estimation $L^{q}-L^{s}$ du semi-groupe (1.6), $U_{0} \in E_{s}$ pour $\left.\left.1 / s \in\right] 0,1 / q\right]$. On a déjà dit que $u \in E_{p}$ et $1 / p<1 / n$ impliquent $\Theta(u, u) \in E_{r}$ pour $1 / r \in] \max \{0,2 / p-1 / n\}, 2 / p]$. Donc $u=U_{0}+\Theta(u, u) \in E_{s}$ pour $1 / s \in[1 / p, \min \{2 / p, 1 / q\}]$. On montre ensuite par récurrence sur $k$ que, pour tout $k$ vérifiant la condition $2^{k} / p \leq 2 / q$, l'assertion : " $u \in E_{s}$ pour tout s vérifiant $1 / s \in\left[1 / p, \min \left\{2^{k} / p, 1 / q\right\}\right]$ " est vraie. Pour $k=1$, la condition est vérifiée puisque $q<p$, et l'assertion est vraie comme on vient de le voir. Pour passer de $k$ à $k+1$, on note que l'assertion au rang $k$ implique $u \in E_{s}$ pour $1 / p \leq 1 / s \leq \min \left\{2^{k} / p, 1 / q\right\}$. La condition au rang $k+1$ implique alors $1 / s \leq 2^{k} / p$ puis $2 / s \leq 2 / q \leq 1 / n^{\prime}$, car $n \leq q$. On peut donc appliquer à nouveau la Proposition 29. Quand la récurrence s'arrête, on obtient $k=K$ tel que l'assertion soit vraie au rang $K$ et $2^{K} / p>1 / q$, donc $u \in E_{s}$ pour $1 / s \in[1 / p, 1 / q]$. Comme $2 / q \leq 1 / n^{\prime}$, on applique une dernière fois la Proposition 29 qui donne $\Theta(u, u) \in E_{s}$ pour $1 / s \in[1 / p, 2 / q]$. Voir sur la Figure 1 la flèche issue de $1 / p_{2}$. 


\subsubsection{Preuve des théorèmes d'existence.}

Lemme 39. Soit $u_{0} \in X_{n}$. Alors

1) $U(t) u_{0} \in E_{p}$ pour $n \leq p$, avec $\left\|U(t) u_{0}\right\|_{E_{p}} \leq C\left\|u_{0}\right\|_{n}$.

2) $u_{0} \in \dot{\mathcal{B}}_{p}$ pour $n \leq p$ avec $\left\|u_{0} \mid \dot{\mathcal{B}}_{p}\right\| \leq C\left\|u_{0}\right\|_{n}$. $E_{p ; 0}(0)$.

3) $\lim _{t \rightarrow 0} t^{[p]}\left\|U(t) u_{0}\right\|_{p}=0$ pour $n<p$, autrement dit $U(t) u_{0} \in$

DÉmonstration. La première assertion découle des inégalités $L^{n}-L^{p}$.

La seconde assertion est une conséquence des inégalités $L^{n}-L^{p}$, de l'estimation uniforme de $t A U(t)$ sur $X_{n}$ et de la définition de $\dot{B}_{p \infty}^{s}(A)$ par (1.14).

La dernière assertion s'obtient par un raisonnement classique : si $u_{0} \in W_{n}^{2}(A)$, alors $\left\|(U(\varepsilon)-\mathrm{Id}) u_{0}\right\|_{n} \leq \varepsilon\left\|A u_{0}\right\|_{n}$. Donc

$$
\begin{aligned}
t^{[p]}\left\|(U(t+\varepsilon)-U(t)) u_{0}\right\|_{p} & \leq t^{[p]}\left\|U(t)(U(\varepsilon)-\mathrm{Id}) u_{0}\right\|_{p} \\
& \leq C\left\|(U(\varepsilon)-\mathrm{Id}) u_{0}\right\|_{n} \leq C \varepsilon\left\|A u_{0}\right\|_{n}
\end{aligned}
$$

donc $t^{[p]}\left\|(U(t+\varepsilon)-U(t)) u_{0}\right\|_{p} \longrightarrow 0$ quand $\varepsilon \longrightarrow 0$, uniformément par rapport à $t$. D'autre part,

$$
t^{[p]}\left\|U(t+\varepsilon) u_{0}\right\|_{p} \leq\left(\frac{t}{t+\varepsilon}\right)^{[p]}\left\|u_{0}\right\|_{n}
$$

Pour tout $\varepsilon>0$ cela tend vers 0 quand $t \longrightarrow 0$, car $[p]>0$ pour $p>n$. En additionnant, $\lim _{t \rightarrow 0} t^{[p]}\left\|U(t) u_{0}\right\|_{p}=0$ pour tout $u_{0} \in W_{n}^{2}(A)$. Or ${ }^{t}{ }^{[p]} U(t)$ est borné dans $\mathcal{L}\left(X_{n}, X_{p}\right)$, et $W_{n}^{2}(A)$ est dense dans $X_{n}$. Donc la convergence a lieu pour tout $u_{0} \in X_{n}$.

DÉmonstration du Théorème 23. Soit $u_{0} \in X_{n}$. D'après le Lemme $39, U_{0} \in E_{q}$ pour $q \geq n$ et $U_{0} \in E_{q ; 0}(0)$ pour $q>n$. De plus on dispose de $p>n$ tel que

$$
\left\|u_{0} \mid \dot{\mathcal{B}}_{p}\right\| \simeq \sup _{t>0} t^{[p]}\left\|U_{0}\right\|_{p}=\left\|U_{0}\right\|_{E_{p}} \leq \eta(p) \doteq\left(4 C_{p}\right)^{-1}
$$

où l'équivalence des normes vient du Lemme 19 et où $C_{p}$ est la constante de continuité de $\Theta: E_{p} \times E_{p} \longrightarrow E_{p}$ fournie par la Proposition 29. D'après la Proposition 36, il existe une solution $u \in E_{p ; 0}(0)$ de $(2.2)$, 
et $\|u\|_{E_{p}} \leq 2\left\|U_{0}\right\|_{E_{p}}$. C'est l'unique solution dans la boule de $E_{p}$ de rayon $2 \eta(p)$.

La Proposition 38 assure que $u \in E_{q}$ pour $q \geq n$ et $u \in E_{q ; 0}(0)$ pour $q>n$. On en déduit que $\Theta(u, u) \in E_{n ; 0}$. Comme par ailleurs on sait que $\lim _{t \rightarrow 0} U_{0}=u_{0}$ dans $X_{n}$, on a bien $u \in C_{b}\left(\overline{\mathbb{R}}_{+}, X_{n}\right)$ et $u(0)=u_{0}$.

DÉmonstration du Théorème 25. Soit $u_{0} \in \dot{\mathcal{B}}_{r}$ et $r>n$. Par la Proposition 38 et le Lemme $19, U_{0}=U(t) u_{0} \in E_{q}$ pour $q \geq r$. De plus on dispose comme ci-dessus de $p \geq r$ tel que

$$
\left\|u_{0} \mid \dot{\mathcal{B}}_{p}\right\| \simeq \sup _{t>0} t^{[p]}\left\|U_{0}\right\|_{p}=\left\|U_{0}\right\|_{E_{p}} \leq \eta(p) \doteq\left(4 C_{p}\right)^{-1}
$$

Donc il existe une solution $u \in E_{p}$ de l'équation (2.2), unique dans la boule de $E_{p}$ de rayon $2 \eta(p)$, et $\|u\|_{E_{p}} \leq 2\left\|U_{0}\right\|_{E_{p}}$.

La Proposition 38 assure que $u \in E_{q}$ pour $q \geq r$. D'après les propositions 30 et $28, \Theta(u, u) \in C_{b}\left(\mathbb{R}_{+} ; \dot{\mathcal{B}}_{r}\right)$ et $\Theta(u, u)(t) \longrightarrow 0$ dans $W_{r}^{-2}(A)$ quand $t \longrightarrow 0$. Or $U_{0} \in C_{b}\left(\mathbb{R}_{+} ; \dot{\mathcal{B}}_{r}\right)$ et $U_{0} \longrightarrow u_{0}$ dans $W_{r}^{-2}(A)$ quand $t \longrightarrow 0$, d'après le Lemme 21 . Donc $u$ aussi.

\subsubsection{Quand $t \longrightarrow \infty$.}

Ayant des solutions globales, on s'intéresse à leur comportement quand $t$ tend vers l'infini. Nous avons introduit les espaces $E_{p ; 0}(\infty)$ dans ce but. Comme [28] dans le cas de $\mathbb{R}^{3}$, nous montrons que deux solutions ont le même comportement à l'infini s'il en va de même de leurs parties linéaires.

Théorème 40. Soit $n<p$, et $u^{1}, u^{2}$ deux solutions dans $E_{p}$ de l'équation de point fixe

$$
u^{1}=U_{0}^{1}-\Theta\left(u^{1}, u^{1}\right), \quad u^{2}=U_{0}^{2}-\Theta\left(u^{2}, u^{2}\right) .
$$

Étant donné $n \leq q$, on suppose que $\left\|u^{1}\right\|_{E_{p}}+\left\|u^{2}\right\|_{E_{p}}<C^{-1}$ où $C$ est la constante de continuité de $\Theta$ de $E_{p} \times E_{q}$ où $E_{q} \times E_{p}$ dans $E_{q}$. Si $U_{0}^{2}-U_{0}^{1} \in E_{q ; 0}(\infty)$ alors $u^{2}-u^{1} \in E_{q ; 0}(\infty)$.

DÉmonstration. Notons $w=u^{2}-u^{1}$ et $W_{0}=U_{0}^{2}-U_{0}^{1}$. Alors $w=W_{0}-\Theta\left(w, u^{2}\right)-\Theta\left(u^{1}, w\right)$. Cette équation de point fixe $w=g(w)$ 
a un sens dans $E_{q}$ dès que $W_{0} \in E_{q}$ et $\Theta$ est continu de $E_{p} \times E_{q}$ et $E_{q} \times E_{p}$ dans $E_{q}$. Elle admet une solution unique puisque $g$ est affine et contractante de rapport $k=C\left(\left\|u^{1}\right\|_{E_{p}}+\left\|u^{2}\right\|_{E_{p}}\right)<1$. w est nécessairement la limite dans $E_{q}$ de la suite définie par $w_{0}=W_{0}$, $w_{i+1}=g\left(w_{i}\right)$. On montre alors par récurrence que $w_{i} \in E_{q ; 0}(\infty)$ dès que $W_{0} \in E_{q ; 0}(\infty)$, grâce à la Proposition 29. Il s'ensuit que $w \in$ $E_{q ; 0}(\infty)$.

\subsection{Unicité des solutions locales $C\left(X_{n}\right)$.}

Lemme 41. Soit $u_{0} \in X_{n}$ et $U_{0} \doteq U(t) u_{0}$. Soit $u^{1}$ et $u^{2}$ dans $C\left(\left[0, T\left[; X_{n}\right)\right.\right.$, solutions de l'équation $(2.2)$. Alors il existe $\varepsilon>0$ tel que $u^{1}=u^{2}$ sur $[0, \varepsilon] \cap[0, T[$.

DÉmonstration. La différence $w \doteq u^{2}-u^{1}$ des deux solutions de (2.2) vérifie, au moins formellement,

$$
\begin{aligned}
w & =\Theta\left(u^{1}, u^{1}\right)-\Theta\left(u^{2}, u^{2}\right) \\
& =-\Theta\left(w, u^{1}-U_{0}\right)-\Theta\left(u^{2}-U_{0}, w\right)-\Theta\left(w, U_{0}\right)-\Theta\left(U_{0}, w\right) .
\end{aligned}
$$

Par le Corollaire 34, on sait que $\Theta$ est bilinéaire continu de $G_{r}^{\varepsilon} \times F_{p}^{\varepsilon}$ et $F_{p}^{\varepsilon} \times G_{r}^{\varepsilon}$ dans $G_{r}^{\varepsilon}$, pour $r$ et $p$ vérifiant (3.8), avec une norme indépendante de $\varepsilon \leq 1$. Fixons $p$ et $r$ tels que $0<1 / r-1 / n<1 / p<$ $1 / n$. On a alors

$$
\|w\|_{G_{r}^{\varepsilon}} \leq C\|w\|_{G_{r}^{\varepsilon}}\left(2\left\|U_{0}\right\|_{F_{p}^{\varepsilon}}+\left\|u^{1}-U_{0}\right\|_{F_{n}^{\varepsilon}}+\left\|u^{2}-U_{0}\right\|_{F_{n}^{\varepsilon}}\right) .
$$

Le premier terme dans la parenthèse tend vers 0 quand $\varepsilon \longrightarrow 0$, d'après le Lemme 39. Les deux autres termes tendent aussi vers 0 car $u^{i}$ et $U_{0}$ sont continues à valeurs dans $X_{n}$ et égales en 0 . Pour $\varepsilon$ assez petit, $\|w\|_{G_{r}^{\varepsilon}}<\|w\|_{G_{r}^{\varepsilon}}$ et donc $w=0$ sur $[0, \varepsilon]$.

DÉmonstration Du Théorème 26. Soit $J \doteq\left\{t \in\left[0, T\left[: u^{1}=\right.\right.\right.$ $u^{2}$ sur $\left.[0, t]\right\}$. Par continuité des fonctions $u^{i}, J$ est fermé. Pour tout $T^{\prime} \in\left[0, T\left[, \widetilde{u}^{i}: t \longmapsto u^{i}\left(T^{\prime}+t\right)\right.\right.$ appartient à $C\left(\left[0, T-T^{\prime}\left[; X_{n}\right)\right.\right.$, et résout l'équation (2.2) pour la donnée initiale $\widetilde{u}_{0}^{i}=u^{i}\left(T^{\prime}\right)$. Le lemme appliqué à $\widetilde{u}^{i}$ assure que $J$ est ouvert. Or $0 \in J$ car $u^{1}(0)=u_{0}=u^{2}(0)$, donc $J=[0, T[$. 


\section{L'exemple.}

Il s'agit de démontrer le Lemme 24 en exhibant un exemple de suite $u^{k}$. Comme dans [4], nous allons montrer deux points. D'une part que le produit de $u_{0}$ par une suite $w^{k}$ très oscillante tend vers 0 dans un espace de Besov homogène d'indice négatif. D'autre part qu'en choisissant convenablement $w^{k}$, le produit ne tend pas vers 0 en norme $L^{p}$. Plus précisément, voici les énoncés qui tiennent compte du fait que le produit $u_{0} w^{k}$ n'est pas en général dans $X_{n}$ :

Proposition 42. Soit $u_{0} \in L^{n}(\Omega)$ et $\left\{w^{k}: k \in \mathbb{N}\right\} \subset L^{\infty}(\Omega)$ une suite bornée qui tend faiblement vers 0 . Alors pour $n<p$

$$
\lim _{k \rightarrow \infty} \sup _{t>0} t^{[p]}\left\|U(t) P\left(u_{0} w^{k}\right)\right\|_{p}=0 .
$$

Proposition 43. Soit $u_{0} \in L^{n}(\Omega)$ et $w^{k}(x)=\phi(x) \exp (i k \xi \cdot x)$, où $\phi \in C^{\infty}(\Omega)$ est nulle au voisinage de $\partial \Omega$ et vaut 1 au voisinage de $\infty$ et $\xi \in \mathbb{R}^{n}$ avec $|\xi|=1$. Si $\left\|\phi u_{0}\right\|_{p}>0$ alors on peut choisir $\xi$ de sorte que

$$
\liminf _{k \in \mathbb{N}}\left\|P\left(u_{0} w^{k}\right)\right\|_{p}>0 \text {. }
$$

\subsection{Convergence dans un espace de Besov d'indice négatif.}

La preuve de la Proposition 42 consiste à se ramener au

Lemme 44. Soit $u_{1} \in C_{0}^{\infty}(\Omega)$ et $\left\{w^{k}\right\}$ comme dans l'énoncé de la proposition. Soit $K$ un compact de $\mathbb{C} \backslash]-\infty, 0]$. Pour tout $\mu \in K$,

$$
\lim _{k \rightarrow \infty}\left\|(\mu+A)^{-1} P\left(u_{1} w^{k}\right)\right\|_{p}=0 .
$$

Preuve que le Lemme 44 implique la Proposition 42. Fixons $\varepsilon>0$. On peut toujours écrire $u_{0}=u_{1}+u_{2}$ avec $u_{1} \in C_{0}^{\infty}(\Omega)$ et $\left\|u_{2}\right\|_{n}<\varepsilon$, et donc, d'après (1.6),

$$
\sup _{k \in \mathbb{N}} \sup _{t>0} t^{[p]}\left\|U(t) P\left(u_{2} w^{k}\right)\right\|_{p} \leq C \sup _{k \in \mathbb{N}}\left\|P\left(u_{2} w^{k}\right)\right\|_{n} \leq C \varepsilon .
$$

Pour $u_{1}$, on dit d'abord qu'il existe $T_{\varepsilon}$ tel que

$$
\sup _{k \in \mathbb{N}} \sup _{t \notin\left[T_{\varepsilon}^{-1}, T_{\varepsilon}\right]} t^{[p]}\left\|U(t) P\left(u_{1} w^{k}\right)\right\|_{p} \leq C \varepsilon .
$$


En effet, pour $t<T_{\varepsilon}^{-1}$, on invoque que $u_{1}$ appartient à $L^{p}(\Omega)$, donc la suite des $P\left(u_{1} w^{k}\right)$ est bornée dans $X_{p}$. Comme le semi-groupe $U(t)$ est borné sur cet espace, il ne reste plus qu'à majorer $t^{[p]}$. Or pour $p>n$, on sait que $[p]>0$. Pour $t>T_{\varepsilon}$, on tient le même raisonnement en partant de $u_{1} \in L^{q}(\Omega)$, en choisissant $q<n$. Notons simplement que $[p]-[q, p]=[q]<0$.

Sur l'intervalle compact $\left[T_{\varepsilon}^{-1}, T_{\varepsilon}\right]$ les fonctions

$$
t \longmapsto t^{[p]}\left\|U(t) P\left(u_{1} w^{k}\right)\right\|_{p}
$$

sont uniformément équicontinues. En effet on sait qu'il existe des constantes telles que

$$
\partial_{t} U(t) u=-A U(t) u \quad \text { et } \quad \sup _{t>0}\|t A U(t) u\|_{p} \leq C\|u\|_{p},
$$

pour tout $u \in X_{p}$,

$$
\sup _{t>0}\left\|t^{1+[p]} A U(t) u\right\|_{p} \leq C\|u\|_{n}
$$

pour tout $u \in X_{n}$. En appliquant ceci à $u=u_{1} w^{k}$, on obtient

$$
\sup _{k \in \mathbb{N}}\left\|\partial_{t}\left(t^{[p]} U(t) P\left(u_{1} w^{k}\right)\right)\right\|_{p} \leq C t^{-1}\left\|u_{1}\right\|_{n}
$$

pour tout $t \in\left[T_{\varepsilon}^{-1}, T_{\varepsilon}\right]$. La norme $\|\cdot\|_{p}$ est une application lipschitzienne. On en déduit l'équicontinuité uniforme des fonctions

$$
t^{[p]}\left\|U(t) P\left(u_{1} w^{k}\right)\right\|_{p} .
$$

D'après l'une des versions du théorème d'Ascoli, une suite équicontinue de fonctions qui converge simplement converge uniformément sur tout compact. Il nous suffit donc de vérifier que pour tout $t \in\left[T_{\varepsilon}^{-1}, T_{\varepsilon}\right]$,

$$
\lim _{k \rightarrow \infty}\left\|U(t) P\left(u_{1} w^{k}\right)\right\|_{p}=0
$$

On fixe donc maintenant $t$ dans cet intervalle.

Il est plus facile de contrôler l'influence du bord $\partial \Omega$ sur la résolvante. On écrit donc le semi-groupe à l'aide de l'intégrale de Dunford sur le contour $\Gamma=\Gamma_{\theta}$, qui est le bord dans $\left.\left.\mathbb{C} \backslash\right]-\infty, 0\right]$ de l'ouvert contenant 
les $\lambda$ tels que $\pi-\theta<|\arg (\lambda)|<\pi$ ou $|\lambda|<\theta$ (où on a fixé un $\theta$ tel que $\pi / 2<\pi-\theta<\pi)$

$$
\begin{aligned}
U(t) u & =\frac{1}{2 i \pi} \int_{\Gamma} e^{t \lambda}(\lambda+A)^{-1} u d \lambda \\
& =\frac{1}{2 i \pi} \int_{\Gamma} e^{\lambda} t^{-1}\left(t^{-1} \lambda+A\right)^{-1} u d \lambda
\end{aligned}
$$

La seconde égalité vient du changement de variable $t \lambda \longmapsto \lambda$. On peut conserver le même contour $\Gamma$ d'intégration grâce au théorème de Cauchy. On sait grâce l'estimation (1.4) sur la résolvante que

$$
\sup _{k \in \mathbb{N}}\left\|(\lambda+A)^{-1} P\left(u_{1} w^{k}\right)\right\|_{p} \leq C|\lambda|^{-1}\left\|u_{1}\right\|_{p} .
$$

Il existe donc $R_{\varepsilon}$ tel qu'en notant $B_{\varepsilon}$ la boule fermée dans $\mathbb{C}$ de rayon $R_{\varepsilon}$, on ait

$$
\sup _{k \in \mathbb{N}}\left\|\frac{1}{2 i \pi} \int_{\Gamma \backslash B_{\varepsilon}} e^{\lambda} t^{-1}\left(t^{-1} \lambda+A\right)^{-1} P\left(u_{1} w^{k}\right) d \lambda\right\|_{p} \leq C \varepsilon .
$$

Appelons $K$ le compact de $\mathbb{C} \backslash]-\infty, 0]$ que décrit $t^{-1} \lambda$ quand $t$ varie dans $\left[T_{\varepsilon}^{-1}, T_{\varepsilon}\right]$ et $\lambda$ varie dans $\Gamma \cap B_{\varepsilon}$. Grâce au théorème de convergence dominée appliquée à l'intégrale sur $\Gamma \cap B_{\varepsilon}$, il nous suffit donc de montrer que pour tout $\mu \in K$,

$$
\lim _{k \rightarrow \infty}\left\|(\mu+A)^{-1} P\left(\mu_{1} w^{k}\right)\right\|_{p}=0 .
$$

Nous décomposons la démonstration du Lemme 44 en trois étapes qui s'enchaînent naturellement.

Lemme 45 (Première étape). Soit $u_{1} \in C_{0}^{\infty}(\Omega)$ et $\left\{w^{k}\right\}$ une suite bornée dans $L^{\infty}(\Omega)$ qui tend vers 0 pour la topologie $\star$-faible. Alors $f^{k}=P\left(u_{1} w^{k}\right)$ est bornée dans $X_{p}$, tend faiblement vers 0 , et est uniformément p-intégrable à l'infini: pour tout $\varepsilon$ il existe $R_{\varepsilon}$ tel que pour tout $k \in \mathbb{N}$

$$
\left\|f^{k}\right\|_{p, \Omega \backslash B_{\varepsilon}}<\varepsilon
$$

où $B_{\varepsilon}$ est la boule fermée de rayon $R_{\varepsilon}$. 
DÉmonstration. La borne dans $X_{p}$ et la convergence faible viennent de la continuité (forte et donc aussi faible) de $P$.

Soit $K^{\prime}$ un compact de $\Omega$ contenant le support de $u_{1}$. Pour tout $u$ dans $L^{p}\left(K^{\prime}\right)$ étendu à $\Omega$ par 0 hors de $K^{\prime}$, on sait que $(1-P) u=\nabla p$ où $p$ est l'unique solution du problème de Laplace-Neumann

$$
\Delta p=\nabla \cdot u, \quad \gamma_{1} p=0, \quad \nabla p \in L^{p}(\Omega) .
$$

En effet, d'après la décomposition de Helmholtz (1.1), il existe un $p$ dans $L_{\text {loc }}^{p}(\Omega)$, unique à une constante près, tel que $(1-P) u=\nabla p$. Ceci implique que $p$ vérifie $\Delta p=\nabla \cdot u$ et $\gamma_{1} p=0$, puisque le support de $u$ ne rencontre pas $\partial \Omega$. L'unicité de $p$ sous la condition $\nabla p \in L^{p}(\Omega)$ vient de [25, Lemma 1.4]. Soit $B$ une boule de rayon $R$ si grand que $\left(K^{\prime} \cup \partial \Omega\right) \subset(1 / 2) B$. Il est clair que $p$ et donc $\nabla p$ sont harmoniques sur $\Omega \backslash B$. D'après le Théorème 50 de représentation des fonctions harmoniques, on peut développer $p$ en série de Laurent

$$
p(x)=\sum_{j=0}^{\infty}|x|^{2-n-2 j} H_{j}(x)+\sum_{j=0}^{\infty} H_{j}^{\prime}(x)
$$

où $H_{j}$ et $H_{j}^{\prime}$ sont des polynômes harmoniques homogènes de degré $j$. La première série converge uniformément sur $\Omega \backslash B$, tandis que la seconde converge uniformément sur les couronnes $B_{r} \backslash B$ pour tout $r \geq R$, avec $B_{r}$ la boule de rayon $r$. La série est dérivable terme à terme

$$
\nabla p(x)=\sum_{j=0}^{\infty} \nabla\left(|x|^{2-n-2 j} H_{j}(x)\right)+\sum_{j=0}^{\infty} \nabla H_{j}^{\prime}(x) .
$$

Le terme $\nabla H_{0}^{\prime}$ est nul, et comme on sait que $\nabla p \in L^{p}(\Omega)$, nécéssairement $H_{j}^{\prime}(x)=0$ pour $j>0$. Dans la première série, le terme d'indice $j$ est en $O\left(|x|^{1-n-j}\right)$, et le terme d'indice 0 est exactement $c_{n} H_{0}|x|^{-n} x$. Par ailleurs le théorème de Stokes implique, pour tout $r \geq R$,

$$
\int_{\partial B_{r}} \gamma_{1} p=\int_{\Omega \cap B_{r}} \nabla \cdot u-\int_{\partial \Omega} \gamma_{1} p=\int_{\partial \Omega \cup \partial B_{r}} \gamma_{\nu} u=0 .
$$

Si on remplace $p$ par la série dans l'intégrale, quand on fait tendre $r$ vers l'infini, on voit que nécessairement $H_{0}(x)=0$. On obtient $\nabla p(x)=$ $O\left(|x|^{-n}\right)$ quand $x \longrightarrow \infty$. Notons $\mathcal{H}$ l'espace des champs harmoniques sur $\Omega \backslash B$, continus sur $\overline{\Omega \backslash B}$ et telles que

$$
\|u\|_{\mathcal{H}} \doteq \sup _{x \in \Omega \backslash B}|x|^{n}|u(x)|<\infty .
$$


Il est immédiat que $\mathcal{H}$ muni de $\|u\|_{\mathcal{H}}$ est un espace de Banach. On considère l'opérateur linéaire $Q$ de $L^{p}\left(K^{\prime}\right)$ dans $\mathcal{H}$ qui associe à $u$ la restriction à $\Omega \backslash B$ de $u-P u=\nabla p$. Nous affirmons que $Q$ est un opérateur fermé. En effet soit $u^{k} \longrightarrow u$ dans $L^{p}\left(K^{\prime}\right)$ et $Q u^{k} \longrightarrow v$ dans $\mathcal{H}$. Ceci implique en particulier que $Q u^{k} \longrightarrow v$ dans $L^{p}(\Omega \backslash B)$. Par ailleurs, la continuité de $1-P$ sur $L^{p}(\Omega)$ implique que $Q u^{k} \longrightarrow Q u$ dans $L^{p}(\Omega \backslash B)$. Donc $v=Q u$. Par le théorème du graphe fermé, on a donc une constante $C$ telle que pour tout $u \in L^{p}\left(K^{\prime}\right)$,

$$
\|Q u\|_{\mathcal{H}} \leq C\|u\|_{p, K^{\prime}} .
$$

Revenons à $f^{k}=P\left(u_{1} w^{k}\right)=u_{1} w^{k}-(1-P)\left(u_{1} w^{k}\right)$. On voit que

$$
\sup _{x \in \Omega \backslash B}|x|^{n}\left|f^{k}(x)\right| \leq C\left\|u_{1}\right\|_{p, K^{\prime}} \text {. }
$$

Cela implique bien que $f^{k}$ est uniformément $p$-intégrable à l'infini.

Avant d'énoncer le deuxième point, rappelons que $\widetilde{E}_{\lambda}$ a été défini à la Section 1.2.1 pour $\lambda \in \mathbb{C} \backslash]-\infty, 0]$, comme l'opérateur de convolution par le potentiel volume $\widetilde{e}_{\lambda}$, transformée de Fourier inverse de $\left(\lambda+|\xi|^{2}\right)^{-1}$.

Lemme 46 (Deuxième étape). Soit $\lambda \in K$ et $f^{k}$ une suite bornée dans $X_{p}$, qui tend faiblement vers 0 et est uniformément $p$-intégrable à l'infini (i.e. vérifie (4.2)). On note $\tilde{f}^{k}$ l'extension de $f^{k}$ par 0 hors de $\Omega$, et $\widetilde{u}^{k}=\widetilde{E}_{\lambda} \widetilde{f}^{k}$. Alors $\widetilde{u}^{k}$ est bornée dans $W_{p}^{1}\left(\mathbb{R}^{n}\right)$, à divergence nulle, et tend vers 0 dans $L^{p}\left(\mathbb{R}^{n}\right)$.

DÉmonstration. Il est immédiat que $\widetilde{f}^{k}=\mathrm{e}_{\Omega} f^{k}$ est bornée dans $L^{p}\left(\mathbb{R}^{n}\right)$, et on a déjà vu que $\nabla \cdot \widetilde{f}^{k}=0$. Par le théorème Mihlin, on en déduit que $\widetilde{u}^{k}$ est bornée dans $W_{p}^{1}\left(\mathbb{R}^{n}\right)$ et à divergence nulle. Soit $\chi_{R}=\chi(x / R)$ avec $\chi$ une fonction régulière égale à 1 sur la boule de rayon 1 et nulle hors de la boule de rayon 2. Quel que soit $R>0$, la suite $\chi_{2 R} \widetilde{u}^{k}$ est donc compacte dans $L^{p}\left(\mathbb{R}^{n}\right)$, d'après l'injection de Sobolev. Soit $\phi$ dans $C_{0}^{\infty}\left(\mathbb{R}^{n}\right)$. $\widetilde{E}_{\lambda}$ étant continu sur $L^{p}\left(\mathbb{R}^{n}\right)$, la convergence faible supposée de $f^{k}$ implique que $\widetilde{u}^{k}$ converge faiblement vers 0 dans $L^{p}\left(\mathbb{R}^{n}\right)$, de même que $\chi_{2 R} \widetilde{u}^{k}$. Par compacité, $\chi_{2 R} \widetilde{u}^{k}$ tend fortement vers 0 .

Si $|x|>2 R$, alors

$$
\left(\chi_{R} \widetilde{e}_{\lambda} * \widetilde{f}^{k}\right)(x)=\left(\chi_{R} \widetilde{e}_{\lambda} *\left(1-\chi_{R / 2}\right) \widetilde{f}^{k}\right)(x) .
$$


Donc, d'après (4.2), pour tout $\varepsilon$ il existe $R_{\varepsilon}$ tel que pour tout $R>R_{\varepsilon}$ et tout $k \in \mathbb{N}$,

$$
\left\|\left(1-\chi_{2 R}\right)\left(\chi_{R} \widetilde{e}_{\lambda} * \widetilde{f}^{k}\right)\right\|_{p} \leq C\left\|\left(1-\chi_{R / 2}\right) \widetilde{f}^{k}\right\|_{p}<\varepsilon .
$$

D'un autre côté, comme $\xi \longmapsto \partial_{\xi}^{\alpha}\left(\lambda+|\xi|^{2}\right)^{-1}$ est $L^{1}\left(\mathbb{R}^{n}\right)$ pour $|\alpha|=$ $n+2$, il existe $C$ tel que

$$
\left|\left(1-\chi_{R}\right) \widetilde{e}_{\lambda}(x)\right| \leq C\langle R\rangle^{-1}\langle x\rangle^{-n-1}, \quad\left\|\left(1-\chi_{R}\right) \widetilde{e}_{\lambda}\right\|_{1} \leq C\langle R\rangle^{-1},
$$

et donc, quitte à augmenter $R_{\varepsilon}$, pour tout $R>R_{\varepsilon}$ et tout $k \in \mathbb{N}$,

$$
\left\|\left(1-\chi_{2 R}\right)\left(\left(1-\chi_{R}\right) \widetilde{e}_{\lambda} * \tilde{f}^{k}\right)\right\|_{p} \leq C\langle R\rangle^{-1}\left\|\tilde{f}^{k}\right\|_{p}<\varepsilon .
$$

Finalement on a montré que

$$
\lim _{R \rightarrow \infty} \sup _{k \in \mathbb{N}}\left\|\left(1-\chi_{2 R}\right) \widetilde{u}^{k}\right\|_{p}=0
$$

ce qui implique que $\widetilde{u}^{k}$ tend vers 0 en norme $L^{p}$.

Avant d'énoncer la troisième étape, rappelons la décomposition de la résolvante vue à la Section 1.2.1. Soit $f \in X_{p}$. On note $\widetilde{f}=\mathrm{e}_{\Omega} f$ et $\widetilde{u}=\widetilde{E}_{\lambda} \widetilde{f}$. Alors on peut écrire

$$
\begin{gathered}
(\lambda+A)^{-1} f=u_{\nu}+u_{\tau}=\left(1-V_{\lambda} \gamma_{0}\right) P \mathrm{r}_{\Omega} \widetilde{u} \\
u_{\nu}=P \mathrm{r}_{\Omega} \widetilde{u}=P_{\nu} \mathrm{r}_{\Omega} \widetilde{u}=\left(1-\nabla N \gamma_{\nu}\right) \mathrm{r}_{\Omega} \widetilde{u} \\
u_{\tau}=-V_{\lambda} \gamma_{0} u_{\nu}, \quad \gamma_{0} u_{\nu}=\gamma_{0} \mathrm{r}_{\Omega} \widetilde{u}-\left(\gamma_{0} \nabla N\right) \gamma_{\nu} \mathrm{r}_{\Omega} \widetilde{u},
\end{gathered}
$$

où $V_{\lambda}$ est l'opérateur solution du problème de Stokes tangentiel (1.3).

Lemme 47 (Troisième étape). Soit $\widetilde{u}^{k}$ une suite bornée dans $W_{p}^{1}\left(\mathbb{R}^{n}\right)$, $\grave{a}$ divergence nulle, et qui tend vers 0 dans $L^{p}\left(\mathbb{R}^{n}\right)$. Alors $u^{k}=(1-$ $\left.V_{\lambda} \gamma_{0}\right) P \mathrm{r}_{\Omega} \widetilde{u}^{k}$ tend vers 0 dans $L^{p}(\Omega)$.

DÉmonstration. Il est clair que l'hypothèse implique $\mathrm{r}_{\Omega} \widetilde{u}^{k}$ tend vers 0 dans $L^{p}$. Comme $P$ est continu sur $L^{p}(\Omega)$, la conclusion s'étend à $u_{\nu}^{k}=P \mathrm{r}_{\Omega} \widetilde{u}^{k}$. La borne sur $\nabla \widetilde{u}^{k}$ dans $L^{p}\left(R^{n}\right)$ et l'inégalité de trace

$$
\left\|\gamma_{0} \mathrm{r}_{\Omega} \widetilde{u}\right\|_{p, \partial \Omega} \leq C\|\widetilde{u}\|_{p, \mathbb{R}^{n}}^{1-1 / p}\|\nabla \widetilde{u}\|_{p, \mathbb{R}^{n}}^{1 / p}
$$


montrent que $\gamma_{0} \mathrm{r}_{\Omega} \widetilde{u}^{k}$ tend vers 0 dans $L^{p}(\partial \Omega)$. D'après [9, Lemma 2.3], $\gamma_{0} \nabla N$ est un opérateur continu sur cet espace, donc $\psi^{k}=-\gamma_{0} u_{\nu}^{k}=$ $\left(1-\gamma_{0} \nabla N \pi_{\nu}\right) \gamma_{0} \mathrm{r}_{\Omega} \widetilde{u}^{k}$ tend encore vers 0 dans cet espace. Finalement la continuité de $V_{\lambda}$ de $L^{p}(\partial \Omega)$ dans $L^{p}(\Omega)$ implique que $u_{\tau}^{k}=V_{\lambda} \psi^{k}$ tend vers 0 .

Résumons la preuve du Lemme 44. On écrit $(\lambda+A)^{-1} P\left(u_{1} w^{k}\right)=$ $u^{k}$ avec

$$
\begin{gathered}
u^{k}=\left(1-V_{\lambda} \gamma_{0}\right) P \mathrm{r}_{\Omega} \widetilde{u}^{k}, \\
\widetilde{u}^{k}=\widetilde{E}_{\lambda} \widetilde{f}^{k}, \quad \widetilde{f}^{k}=\mathrm{e}_{\Omega} f^{k}, \\
f^{k}=P\left(u_{1} w^{k}\right),
\end{gathered}
$$

et on applique successivement les lemmes 45, 46 et 47 .

\subsection{Non convergence vers 0 dans un espace de Lebesgue.}

Nous donnons maintenant la preuve de la Proposition 43.

Rappelons tout d'abord un résultat de [4]. On se place sur $\mathbb{R}^{n}$. On note $\widetilde{P}$ l'opérateur de projection sur les champs à divergence nulle. C'est le multiplicateur de Fourier associé à la fonction

$$
\widetilde{P}(\xi)=\left(1-\frac{\xi \otimes \xi}{|\xi|^{2}}\right),
$$

régulière hors de 0 et homogène de degré 0 . Soit $\widetilde{u} \in L^{p}\left(\mathbb{R}^{n}\right)$ pour $1<p<\infty$, et $\widetilde{w}^{k}=e^{i k \xi \cdot x}$ avec $|\xi|=1$. Alors

$$
\lim _{k \rightarrow \infty}\left\|\widetilde{P}\left(\widetilde{u} \widetilde{w}^{k}\right)-\widetilde{w}^{k} \widetilde{P}(\xi) \widetilde{u}\right\|_{p}=0 .
$$

Revenons à la situation de la Proposition 43, $u_{0} w^{k}=u_{0} \phi \exp (i k \xi \cdot x)$. On peut considérer $\widetilde{u}=u_{0} \phi$ comme un fonction dans $L^{n}\left(\mathbb{R}^{n}\right) . \widetilde{P}(\xi) \widetilde{u}$ vaut alors $\phi \widetilde{P}(\xi) u_{0}$. On obtient

$$
\lim _{k \rightarrow \infty}\left\|\widetilde{P}\left(u_{0} w^{k}\right)-w^{k} \widetilde{P}(\xi) u_{0}\right\|_{p}=0 .
$$

Comme $|\exp (i k \xi \cdot x)|=1$, on sait que $\left\|w^{k} \widetilde{P}(\xi) u_{0}\right\|_{p}=\left\|\phi \widetilde{P}(\xi) u_{0}\right\|_{p}$, qui ne dépend pas de $k$ et qu'on peut supposer être non nul en choisissant bien $\xi$, puisque par hypothèse $\left\|\phi u_{0}\right\|_{p}>0$. On en déduit, d'après (4.5), que $\left\|\mathrm{r}_{\Omega} \widetilde{P}\left(u_{0} w^{k}\right)\right\|_{p}$ ne tend pas vers 0 . 
Soit $\mathcal{V}_{\partial \Omega}$ un voisinage de $\partial \Omega$, compact dans $\mathbb{R}^{n}$ et qui ne rencontre pas le support de $\phi$. Il est clair que la restriction de $\phi \widetilde{P}(\xi) u_{0}$ à $\mathcal{V}_{\partial \Omega}$ est nulle, ce qui implique $\left\|\phi \widetilde{P}(\xi) u_{0}\right\|_{p, \mathcal{V}_{\partial \Omega}}=0$. D'après (4.5), on en déduit que $\left\|\mathrm{r}_{\Omega} \widetilde{P}\left(u_{0} w^{k}\right)\right\|_{p, \mathcal{V}_{\partial \Omega} \cap \Omega}$ tend vers 0 . Ceci va nous permettre de passer de $\mathrm{r}_{\Omega} \widetilde{P}\left(u_{0} w^{k}\right)$ à $P\left(u_{0} w^{k}\right)$.

En effet pour un champ $f \in L^{p}(\Omega)$ à divergence nulle, on a déjà vu que $P f=P_{\nu} f=\left(1-\nabla N \gamma_{\nu}\right) f$. Et on a déjà vu que $\gamma_{\nu}$ est continu de l'ensemble des champs à divergence nulle de $L^{p}(\Omega)$ dans $W_{p}^{-1 / p}(\partial \Omega)$. Il est clair que $\gamma_{\nu} u$ ne dépend que de la restriction de $u$ à un voisinage de $\partial \Omega$, donc en fait $\gamma_{\nu}$ est continu de l'ensemble des champs à divergence nulle de $L^{p}\left(\mathcal{V}_{\partial \Omega} \cap \Omega\right)$ dans $W_{p}^{-1 / p}(\partial \Omega)$. Donc $\gamma_{\nu} \mathrm{r}_{\Omega} \widetilde{P}\left(u_{0} w^{k}\right)$ tend vers 0 dans $W_{p}^{-1 / p}(\partial \Omega)$. Comme en fait il s'agit de la trace normale sur $\partial \Omega$ d'une fonction à divergence nulle sur $\mathbb{R}^{n}$ tout entier, le théorème de Stokes implique que son intégrale sur $\partial \Omega$ est nulle.

Ensuite $\nabla N$ est continu de l'ensemble de fonctions $W_{p}^{-1 / p}(\partial \Omega)$ d'intégrale égale à 0 dans $L^{p}(\Omega)$. Cela montre que $\nabla N \gamma_{\nu} \mathrm{r}_{\Omega} \widetilde{P}\left(u_{0} w^{k}\right)$ tend vers 0 dans $L^{p}(\Omega)$. Enfin,

$$
\liminf _{k \rightarrow \infty}\left\|\mathrm{r}_{\Omega} \widetilde{P}\left(u_{0} w^{k}\right)\right\|_{p}>0
$$

et

$$
\lim _{k \rightarrow \infty}\left\|\nabla N \gamma_{\nu} \mathrm{r}_{\Omega} \widetilde{P}\left(u_{0} w^{k}\right)\right\|_{p}=0
$$

impliquent que $P\left(u_{0} w^{k}\right)=\left(1-\nabla N \gamma_{\nu}\right) \mathrm{r}_{\Omega} \widetilde{P}\left(u_{0} w^{k}\right)$ ne tend pas vers 0 dans $X_{n}$.

\section{A. Interpolation réelle.}

Rappelons deux caractérisations des espaces d'interpolation réelle.

Proposition 48. Soit $\left(A_{i},\|\cdot\|_{i}\right), i=0,1$, deux espaces de Banach qui s'injecte continûment chacun dans un espace vectoriel topologique séparé $\mathcal{A}$. Soit $q \in[1, \infty]$ et $\theta \in] 0,1[$. Les deux définitions suivantes définissent le même espace de Banach avec des normes équivalentes.

1) Soit $\xi_{i}$ deux nombres réels tels que $\xi_{0} \xi_{1}<0$ et $(1-\theta) \xi_{0}+\theta \xi_{1}=$ 0 . On considère l'ensemble des éléments $u \in A_{0}+A_{1}$ qui s'écrivent

$$
u=\int_{0}^{\infty} u(t) \frac{d t}{t}
$$


où l'intégrale converge dans $\mathcal{A}$ et où $u(t)$ est une fonction de $\mathbb{R}_{+}$dans $A_{0} \cap A_{1}$ vérifiant

$$
\left\|t^{\xi_{0}}\right\| u(t)\left\|_{0}\right\|_{q *}+\left\|t^{\xi_{1}}\right\| u(t)\left\|_{1}\right\|_{q *}<\infty
$$

La norme de u est la borne inférieure de l'ensemble des quantités (A.2) pour toutes les fonctions $u(t)$ réalisant (A.1).

2) Soient $\xi_{i}$ deux nombres réels tels que $\xi_{0} \xi_{1}<0$ et $(1-\theta) \xi_{0}+$ $\theta \xi_{1}=0$. On considère l'ensemble des éléments $u \in A_{0}+A_{1} q u i$ s'écrivent

$$
u=u_{0}(t)+u_{1}(t), \quad \text { pour tout } t>0,
$$

où $u_{i}(t)$ est une fonction de $\mathbb{R}_{+}$dans $A_{i}$ et

$$
\left\|t^{\xi_{0}}\right\| u_{0}(t)\left\|_{0}\right\|_{q *}+\left\|t^{\xi_{1}}\right\| u_{1}(t)\left\|_{1}\right\|_{q *}<\infty
$$

La norme de u est la borne inférieure de l'ensemble des quantités (A.4) pour tous les couples de fonctions $\left(u_{0}(t), u_{1}(t)\right)$ réalisant (A.3).

On note $\left(A_{0}, A_{1}\right)_{\theta, q}$ cet espace.

Pour la démonstration de cette proposition, d'autres définitions de ces espaces et l'étude de leurs propriétés, nous renvoyons aux ouvrages tels que [1] ou [29], très complets avec de nombreuses références historiques.

L'intérêt principal de l'interpolation pour les équations aux dérivées partielles réside dans la propriété suivante :

Théorème 49. Soit $\left(A_{i},\|\cdot\|_{i}\right), i=0,1$, deux espaces de Banach qui s'injectent continûment chacun dans un espace vectoriel topologique séparé $\mathcal{A}$. De même $\left(B_{i},\|\cdot\|_{i}\right)$ et $\mathcal{B}$.

Soit $T$ un opérateur linéaire de $A_{0}+A_{1}$ dans $B_{0}+B_{1}$, continu de $A_{0}$ dans $B_{0}$ et de $A_{1}$ dans $B_{1}$.

Alors, pour tout $\theta \in] 0,1\left[\right.$ et $q \in[1, \infty]$, T est continu de $\left(A_{0}, A_{1}\right)_{\theta, q}$ dans $\left(B_{0}, B_{1}\right)_{\theta, q}$ et

$$
\|T\|_{\mathcal{L}\left(\left(A_{0}, A_{1}\right)_{\theta, q} ;\left(B_{0}, B_{1}\right)_{\theta, q}\right)} \leq\|T\|_{\mathcal{L}\left(A_{0} ; B_{0}\right)}^{1-\theta}\|T\|_{\mathcal{L}\left(A_{1} ; B_{1}\right)}^{\theta} .
$$




\section{B. Éléments de théorie du potentiel.}

Soit $\Omega \subset \mathbb{R}^{n}$ un ouvert à bord $\partial \Omega$ lisse. Rappelons que $\gamma_{0}$ est l'opérateur de trace au bord relatif à $\Omega$ : si $u$ est continu sur $\bar{\Omega}, \gamma_{0} u(x)$ est la limite de $u(y)$ quand $y \in \Omega$ tend vers $x \in \partial \Omega$. On oriente le bord $\partial \Omega$ à l'aide du vecteur normal unitaire $\nu$ rentrant. Les opérateurs de trace au bord $\gamma_{\nu}$ et $\gamma_{1}$ sont définis en conséquence, avec en particulier $\gamma_{1}=\nu \cdot \gamma_{0} \nabla=\gamma_{\nu} \nabla$. Les formules de Stokes et Green s'écrivent donc

$$
\begin{aligned}
\int_{\Omega}-\nabla \cdot u & =\int \gamma_{\nu} u \\
\int_{\Omega}(u(-\Delta v)-(-\Delta u) v) & =\int_{\partial \Omega}\left(\gamma_{0} u \gamma_{1} v-\gamma_{1} u \gamma_{0} v\right) .
\end{aligned}
$$

On note $\Omega^{\prime}$ l'ouvert tel que $\mathbb{R}^{n}=\Omega \cup \partial \Omega \cup \Omega^{\prime}$. On note $\gamma_{0}^{\prime}$ l'opérateur de trace de $\bar{\Omega}^{\prime}$ sur $\partial \Omega^{\prime}=\partial \Omega$. Il s'agit de la limite de $u(y)$ quand $y$, dans $\Omega^{\prime}$, tend vers $x \in \partial \Omega$. De même, on définit $\gamma_{\nu}^{\prime}=\nu \cdot \gamma_{0}^{\prime}$ et $\gamma_{1}^{\prime}=\gamma_{\nu}^{\prime} \nabla$. Notons que ces deux opérateurs sont les opposés des opérateurs $\gamma_{\nu}$ et $\gamma_{1}$ relatifs à $\Omega^{\prime}$.

On note $E(x)=c_{n}|x|^{2-n}$ la solution fondamentale de l'équation de Laplace en dimension $n \geq 3:-\Delta E=\delta$, avec $\delta$ la masse de Dirac en 0 . On note $G_{x}$ la fonction de Green associée qui à $y$ fait correspondre $G_{x}(y)=E(x-y)$.

Dans la suite de cette section, on convient que $\nabla G_{x}$ désigne le gradient de la fonction de Green par rapport à sa deuxième variable. De même pour la trace $\gamma_{0} G_{x}$ : c'est la seconde variable qu'on astreint à rester sur $\partial \Omega$. Pour noter qu'une dérivée ou une trace se rapporte à la première variable, on ajoutera un indice : $\nabla_{(x)} G_{x}, \gamma_{0(x)} G_{x}$. En particulier $-\Delta_{(x)} G_{x}=-\Delta G_{x}=\delta_{x}$ où $\delta_{x}$ est la masse de Dirac au point $x$, et

$$
\gamma_{1} G_{x}(y)=-\nu(y) \cdot \nabla E(x-y)=\nu(y) \cdot \nabla E(y-x)=\gamma_{1_{(y)}} G_{y}(x)
$$

\section{B.1. Potentiels simple couche et double couche.}

Soit $u$ une fonction $C^{\infty}$ au voisinage d'un ouvert borné $\Omega$. On suppose de plus que $u$ est harmonique, c'est-à-dire qu'elle vérifie $\Delta u=$ 
0. Alors en remplaçant $v$ par $G_{x}$ dans la formule de Green (B.2), on obtient

$$
u(x)=\int_{\partial \Omega}\left(\gamma_{0} u \gamma_{1} G_{x}-\gamma_{1} u \gamma_{0} G_{x}\right) .
$$

Inversement, donnons-nous un ouvert régulier $\Omega$ et une fonction $\psi$ dans $C_{c}^{\infty}(\partial \Omega)$. On définit les potentiels simple couche $V_{\psi}^{(\mathrm{I})}$ et double couche $V_{\psi}^{(\mathrm{II})} \operatorname{par}$

$$
V_{\psi}^{(\mathrm{I})}=\int_{\partial \Omega} \psi \gamma_{0} G_{x}, \quad V_{\psi}^{(\mathrm{II})}=\int_{\partial \Omega} \psi \gamma_{1} G_{x}
$$

La théorie classique du potentiel dit que les restrictions à $\Omega$ (respectivement $\Omega^{\prime}$ ) de ces deux potentiels sont des fonctions $C^{\infty}$ jusqu'au bord de chacun de ces ouverts.

Le potentiel simple couche est continu à travers la surface, mais pas sa dérivée normale au bord. Elle est donnée par la formule

$$
\begin{aligned}
& \gamma_{1} V_{\psi}^{(\mathrm{I})}(x)=-\frac{1}{2} \psi(x)+\int_{\partial \Omega} \psi \gamma_{1(x)} G_{x}, \\
& \gamma_{1}^{\prime} V_{\psi}^{(\mathrm{I})}(x)=+\frac{1}{2} \psi(x)+\int_{\partial \Omega} \psi \gamma_{1(x)} G_{x} .
\end{aligned}
$$

L'intégrale au second membre est une intégrale impropre. On sait que

$$
\nabla_{(x)} G_{x}(y)=c_{n}(2-n)|x-y|^{1-n} \frac{x-y}{|x-y|}
$$

est homogène de degré $1-n$ en $x-y$ à $x$ fixé. Mais si $x$ et $y$ sont astreints à rester sur $\partial \Omega$, quand $y$ tend vers $x,(x-y) /|x-y|$ tend vers le plan tangent à $x$, orthogonal à $\nu(x)$. Et donc $\gamma_{1(x)} G_{x}(y)$ est en $O\left(|x-y|^{2-n}\right)$. Comme $\partial \Omega$ est de dimension $n-1$, l'intégrale impropre est convergente.

Le potentiel double couche n'est pas continu au travers de la surface $\partial \Omega$. Ses traces intérieure et extérieure sont données par

$$
\begin{aligned}
& \gamma_{0} V_{\psi}^{(\mathrm{II})}(x)=+\frac{1}{2} \psi(x)+\int_{\partial \Omega} \psi \gamma_{1} G_{x} \\
& \gamma_{0}^{\prime} V_{\psi}^{(\mathrm{II})}(x)=-\frac{1}{2} \psi(x)+\int_{\partial \Omega} \psi \gamma_{1} G_{x} .
\end{aligned}
$$


Même remarque sur l'intégrale singulière au second membre.

Pour le cas de la dimension d'espace $n=3$, on trouvera ces formules et d'autres encore dans [23].

\section{B.2. Décomposition en série de Laurent.}

Voici l'équivalent pour les fonctions harmoniques dans $\mathbb{R}^{n}$ de la décomposition en série de Laurent pour les fonctions holomorphes dans $\mathbb{C}$.

Théorème 50. Soit $u \in \mathcal{D}^{\prime}(\mathcal{C})$ une distribution sur la couronne $\mathcal{C}$ définie par $\left\{x \in \mathbb{R}^{n}: \quad r<|x|<R\right\}$. Si $\Delta u=0$ (on dit que u est harmonique) alors on peut représenter u par un unique développement en séries de Laurent

$$
u(x)=\sum_{j \in \mathbb{N}} H_{j}^{\prime}(x)+\sum_{j \in \mathbb{N}}|x|^{-2 j+2-n} H_{j}(x)
$$

où $H_{j}^{\prime}$ et $H_{j}$ sont des polynômes harmoniques homogènes de degré $j$. La première (respectivement la seconde) série converge, ainsi que toutes ses dérivées, uniformément sur les boules de rayon inférieur à $R$ (respectivement hors des boules de rayon supérieur à $r$ ).

IndicAtions pour LA PREuve. Comme $\Delta u=0$, le théorème de régularité elliptique implique qu'en réalité $u$ est $C^{\infty}(\mathcal{C})$. Donnons nous une autre couronne $\mathcal{C}_{1} \doteq\left\{x \in \mathbb{R}^{n}: r_{1}<|x|<R_{1}\right\}$ avec $r<r_{1}<R_{1}<$ R. $u$ est alors $C^{\infty}$ au voisinage de $\mathcal{C}_{1}$. D'après (B.4), pour tout $x \in \mathcal{C}_{1}$,

$$
u(x)=\int_{\partial \mathcal{C}_{1}}\left(\gamma_{0} u \gamma_{1} G_{x}-\gamma_{1} u \gamma_{0} G_{x}\right)=u_{r_{1}}(x)+u_{R_{1}}(x)
$$

où $u_{r_{1}}$ est la fonction donnée par l'intégrale quand on restreint le domaine d'intégration à la composante $|y|=r_{1}$ de $\partial \mathcal{C}_{1}$, et de même avec $R_{1}$. Puisque $\gamma_{0} u(y)$ et $\gamma_{1} u(y)$ sont $C^{\infty}, u_{R_{1}}$ et $u_{r_{1}}$ le sont $\operatorname{sur} \overline{\mathcal{C}}$.

La représentation intégrale de $u_{R_{1}}$ permet d'étendre cette fonction en une fonction harmonique sur la boule $B_{R_{1}}, C^{\infty}$ sur la boule fermée. En décomposant la restriction de $u_{R_{1}}$ à la sphère $S_{R_{1}}=\partial B_{R_{1}}$ en harmoniques sphériques, on obtient

$$
u_{R_{1}}(x)=\sum_{j \in \mathbb{N}} \phi_{j}^{\prime}\left(\frac{x}{R_{1}}\right), \quad|x|=R_{1},
$$


où $\phi_{j}^{\prime}$ est une harmonique sphérique d'ordre $j$ et où la série converge uniformément. Alors la fonction

$$
x \longmapsto \sum_{j \in \mathbb{N}}\left(\frac{|x|}{R_{1}}\right)^{j} \phi_{j}^{\prime}\left(\frac{x}{R_{1}}\right)=\sum_{j \in \mathbb{N}} H_{j}^{\prime}(x), \quad|x| \leq R_{1},
$$

est continue sur la boule fermée, harmonique à l'intérieur (car $|x|^{j} \phi_{j}^{\prime}(x)$ est un polynôme harmonique) et prend les mêmes valeurs au bord que $u_{R_{1}}$. C'est donc elle. On a ainsi obtenu un développement de $u_{R_{1}}$, qui donne la première série de (B.8).

On tient un raisonnement similaire pour $u_{r_{1}}$ sur le complémentaire de la boule de rayon $r_{1}$ : la formulation intégrale assure que la fonction tend vers 0 à l'infini, qu'elle est harmonique sur $|x|>r_{1}$ et $C^{\infty}$ sur $|x| \geq r_{1}$. On décompose sa restriction à $|x|=r_{1}$ en harmoniques sphériques.

$$
u_{r_{1}}(x)=\sum_{j \in \mathbb{N}} \phi_{j}\left(\frac{x}{R_{1}}\right), \quad|x|=R_{1},
$$

où $\phi_{j}$ est une harmonique sphérique d'ordre $j$ et où la série converge uniformément. Alors la fonction

$x \longmapsto \sum_{j \in \mathbb{N}}\left(\frac{|x|}{R_{1}}\right)^{-j+2-n} \phi_{j}\left(\frac{x}{R_{1}}\right)=\sum_{j \in \mathbb{N}}|x|^{-2 j+2-n} H_{j}(x), \quad|x| \leq R_{1}$,

est continue sur $|x| \geq r_{1}$, harmonique sur $|x|>r_{1}\left(\operatorname{car} H_{j}(x)=\right.$ $|x|^{j} \phi_{j}(x)$ est un polynôme harmonique homogène de degré $j$ et donc $|x|^{-2 j+2-n} H_{j}(x)$ est encore harmonique) et prend les mêmes valeurs au bord que $u_{R_{1}}$. C'est donc elle. On a ainsi obtenu le développement de $u_{R_{1}}$ qui donne la seconde série de (B.8).

L'unicité du développement s'obtient en fixant une direction $\xi$. On écrit la série en $x=\rho \xi$. Elle se présente alors comme une série de Laurent en la variable réelle $\rho$. Si on fait varier $\rho$ dans $\mathbb{C}$, la série est convergente sur les compacts de la couronne $r<|\rho|<R$. La somme de la série est une fonction holomorphe, nulle sur la partie de la droite réelle incluse dans cette couronne, donc elle est nulle. L'unicité du développement montre en particulier qu'il ne dépend pas de $r_{1}$ et $R_{1}$.

L'assertion sur les séries dérivées découle de l'application du théorème de Harnack pour les fonctions harmoniques aux sommes partielles des séries. 


\section{B.3. Problème de Neumann extérieur.}

Nous expliquons maintenant ce que signifie résoudre "à l'aide d'un potentiel simple couche" le problème de Neumann pour $\Omega$ domaine extérieur. Il s'agit de résoudre, pour $\phi \in C^{\infty}(\partial \Omega)$,

$$
\Delta w=0, \quad \gamma_{1} w=\phi, \quad \lim _{|x| \rightarrow 0} w(x)=0 .
$$

Pour éviter la difficulté liée au fait que $\Omega$ n'est pas compact, on va se ramener à un problème sur $\partial \Omega$, qui est compact par définition d'un domaine extérieur.

On cherche $w$ sous la forme d'un potentiel simple couche, c'est-àdire qu'on cherche en réalité $\psi$ sur $\partial \Omega$ tel que $w$ définit par $w=V_{\psi}^{(\mathrm{I})}$ (voir (B.5)) soit solution de (B.9). On a déjà vu que pour n'importe quel $\psi$, un tel $w$ est harmonique dans $\Omega$. On montre qu'il tend vers 0 quand $|x|$ tend vers l'infini par une majoration directe puisque $G_{x}(y) \leq$ $C|x-y|^{2-n}$ et que $y$ varie dans $\partial \Omega$.

La condition au bord donne l'équation dont $\psi$ doit être solution

$$
\Pi \psi \doteq \gamma_{1} V_{\psi}^{(\mathrm{I})}=\phi .
$$

L'opérateur $\Pi$ est un opérateur pseudodifférentiel sur $\partial \Omega$. Cela vient de $G_{x}(y)=E(x-y)$ et du fait que la convolution par $E$ est, modulo un opérateur à noyau $C^{\infty}$, un opérateur $Q$ pseudodifférentiel sur $\mathbb{R}^{n}$ paramétrix de $-\Delta$. Ce dernier a un symbole polynômial donc $Q$ possède la propriété de transmission par rapport à toutes les hypersurfaces. Nous affirmons que le symbole principal de $\Pi$ est égal à la fonction constante $-1 / 2$. Cela peut se calculer, en se ramenant par localisation dans des cartes au bord de $\partial \Omega$ au cas du demi-espace. On peut aussi s'en convaincre d'après (B.6), comme nous avons déjà fait remarquer que le noyau de l'intégrale au second membre de (B.6) a une singularité sur la diagonale $x=y$ en $O\left(|x-y|^{2-n}\right.$ ) (et on est sur $\partial \Omega$ de dimension $n-1)$. On en déduit que non seulement l'opérateur $\Pi$ est elliptique, mais en plus que son indice est le même que celui de l'identité, 0.

Pour affirmer que $\Pi$ est inversible, montrons qu'il est surjectif. Il nous suffit de montrer que son transposé ${ }^{t} \Pi$ est injectif. Calculons formellement cet opérateur. Soit $\psi$ et $\psi^{\prime}$ dans $C^{\infty}(\partial \Omega)$.

$$
\begin{aligned}
\left\langle{ }^{t} \Pi \psi, \psi^{\prime}\right\rangle & =\left\langle\psi, \Pi \psi^{\prime}\right\rangle \\
& =\int_{\partial \Omega} \psi(x)\left(-\frac{1}{2} \psi^{\prime}(x)+\int_{\partial \Omega} \psi^{\prime} \gamma_{1(x)} G_{x}\right) d x
\end{aligned}
$$




$$
-\frac{1}{2} \int_{\partial \Omega} \psi \psi^{\prime}+\iint_{\partial \Omega^{2}} \psi(x) \psi^{\prime}(y) \gamma_{1(x)} G_{x}(y) d x d y
$$

d'après (B.6). Or $\gamma_{1(x)} G_{x}(y)=\gamma_{1} G_{y}(x)$ d'après (B.3). Donc en appliquant le théorème de Fubini,

$$
\left\langle{ }^{t} \Pi \psi, \psi^{\prime}\right\rangle=-\frac{1}{2} \int_{\partial \Omega} \psi \psi^{\prime}+\int_{\partial \Omega}\left(\int_{\partial \Omega} \psi \gamma_{1} G_{y}\right) \psi^{\prime}(y) d y=\int_{\partial \Omega} \gamma_{0}^{\prime} V_{\psi}^{(\mathrm{II})} \psi^{\prime}
$$

d'après (B.7). Finalement on a montré que ${ }^{t} \Pi \psi=\gamma_{0}^{\prime} V_{\psi}^{(\mathrm{II})}$.

Alors l'injectivité de ${ }^{t} \Pi$ découle de l'unicité pour le problème de Dirichlet sur l'ouvert borné $\Omega^{\prime}$. En effet, $w^{\prime}=V_{\psi}^{(\mathrm{II})}$ est une fonction harmonique sur $\Omega^{\prime}$, et prend au bord $\partial \Omega^{\prime}=\partial \Omega$ la valeur $\gamma_{0}^{\prime} V_{\psi}^{(\mathrm{II})}$. Si ceci est nul, nécessairement $w^{\prime}=0$, par exemple par le principe du maximum. On a montré que le noyau de ${ }^{t} \Pi$ est réduit à 0 . Donc $\Pi$ est surjectif. Comme son indice est nul, il est bijectif.

Puisque $\Pi$ est un opérateur pseudodifférentiel de degré 0 , elliptique et inversible, son inverse est encore un opérateur pseudodifférentiel de degré 0 . Cela permet de résoudre l'équation $\Pi \psi=\phi$ dans, par exemple, $W_{p}^{-1 / p}(\partial \Omega)$. Le potentiel simple couche $w=V_{\psi}^{(\mathrm{I})}$ a encore un sens, puisqu'on peut le voir comme la convolution de $E$ avec la distribution à support compact $\psi \otimes \delta_{\partial \Omega}$.

\section{References.}

[1] Bergh, J., Löfström, J., Interpolation Spaces, an introduction. SpringerVerlag, 1976.

[2] Borchers, W., Myakawa, T., Algebraic $L^{2}$ decay for Navier-Stokes flows in exterior domains. Acta Math. 165 (1990), 189-227.

[3] Borchers, W., Sohr, H., On the semigroup of the Stokes operator for exterior domains in $L^{q}$-spaces. Math. Z. 196 (1987), 415-425.

[4] Cannone, M., Ondelettes, paraproduits et Navier-Stokes. Diderot Éditeur, Arts et Sciences, 1995.

[5] Cannone, M., Planchon, F., Self-similar solutions for Navier-Stokes equations in $\mathbb{R}^{3}$. Comm. Partial Diff. Equations 21 (1996), 179-193.

[6] Fujita, H., Kato, T., On the Navier-Stokes initial value problem I. Arch. Rational Mech. Anal. 16 (1964), 269-315.

[7] Furioli, G., Lemarié-Rieusset, P.-G., Terraneo, E., Sur l'unicité dans $L^{3}\left(\mathbb{R}^{3}\right)$ des solutions "mild" des équations de Navier-Stokes. C. R. Acad. Sci. Paris Sér. I Math. 325 (1997), 1253-1256. 
[8] Furioli, G., Lemarié-Rieusset, P.-G., Terraneo, E., Unicité dans $L^{3}\left(\mathbb{R}^{3}\right)$ et d'autres espaces limites fonctionnels pour Navier-Stokes. Revista Mat. Iberoamericana 16 (2000), 605-667.

[9] Giga, Y., Analyticity of the semigroup generated by the Stokes operator in $L_{r}$ spaces. Math. Z. 178 (1981), 297-329.

[10] Grisvard, P., Commutativité de deux foncteurs d'interpolation et applications. J. Math. Pures et Appl. 45 (1966), 143-290.

[11] Grubb, G., Nonhomogeneous Navier-Stokes problems in $L_{p}$ Sobolev spaces over interior and exterior domains. Proccedings of Oberwohlfach meeting, 1994. Communiqué personnellement par l'auteur.

[12] Grubb, G., Nonhomogeneous time-dependent Navier-Stokes problems in $L_{p}$ Sobolev spaces. Differential and integral equation 8 (1995), 10131046.

[13] Grubb, G., Solonnikov, V. A., Boundary value problems for the nonstationary Navier-Stokes equations treated by pseudo-differential methods. Math. Scand. 69 (1991), 217-290.

[14] Iwashita, H., $L_{q}-L_{r}$ estimates for solutions of nonstationary Stokes equations in an exterior domain and the Navier-Stokes initial value problem in $L_{q}$ spaces. Math. Ann. 285 (1989), 265-288.

[15] Kato, T., Perturbation theory for linear operators. Springer-Verlag, 1966.

[16] Kato, T., Strong $L^{p}$-solutions of the Navier-Stokes equation in $R^{m}$, with applications to weak solutiona. Math. Z. 187 (1984), 471-480.

[17] Kobayashi, T., Muramatu, T., Abstract Besov space approach to the non-stationary Navier-Stokes equations. Math. Methods Appl. Sci. 15 (1992), 599-620.

[18] Kozono, H., Yamazaki, M., Semilinear heat equations and the NavierStokes equation with distributions in new functions spaces as initial data. Comm. Partial Diff. Equations 19 (1994), 959-1014.

[19] Kozono, H., Yamazaki, M., Local and global unique solvability of the Navier-Stokes exterior problem with Cauchy data in the space $L^{n, \infty}$. Houston J. Math. 21 (1995), 755-799.

[20] Lions, J.-L., Peetre, J., Sur une classe d'espaces d'interpolation. Inst. Hautes Études Sci. Publ. Math. 19 (1964), 5-68.

[21] Lions, P.-L., Masmoudi, N., Unicité des solutions faibles de NavierStokes dans $L^{N}(\Omega)$. C. R. Acad. Sci. Paris Sér. I Math. 327 (1998), 491-496.

[22] Maremonti, P., Solonnikov, V. A., On nonstationary Stokes problem in exterior domains. Ann. Scuola Norm. Sup. Pisa Cl. Sci. 24 (1998), 395-450. 
[23] Martensen, E., Potentialtheorie. B. G. Teubner, 1968.

[24] Meyer, Y., Wawelets, paraproducts and Navier-Stokes equations. International Press, 1997.

[25] Myakawa, T., On non stationary solutions of the Navier-Stokes equations in exterior domains. Hiroshima Math. J. 12 (1982), 115-140.

[26] Oru, F., Thèse de doctorat, 1998, École Polytechnique.

[27] Planchon, F., Solutions globales et comportement asymptotique pour les équations de Navier-Stokes. Thèse de doctorat, 1996, École Polytechnique.

[28] Planchon, F., Asymptotic behavior of global solutions to the NavierStokes equations in $\mathbb{R}^{3}$. Revista Mat. Iberoamericana 14 (1998), 71-93.

[29] Triebel, H., Interpolation theory, function spaces, differential operators. North-Holland Publ. Co., 1978.

[30] Triebel, H., Theory of function spaces. Birkhäuser, 1983.

[31] van Neerven, J., The adjoint of a Semigroup of Linear Operators. Springer-Verlag, 1992.

[32] Weissler, F., The Navier-Stokes initial value problem in $L^{p}$. Arch. $R a$ tional Mech. Anal. 74 (1980), 219-230.

Recibido: 24 de febrero de 1.999

Recibido: 15 de septiembre de 1.999

Nicolas Depauw

Mathématiques

Université de Nantes

2, rue de la Houssinière

BP 92208

44322 Nantes Cedex 3, FRANCE

Nicolas . Depauw@math . univ-nantes .fr 